\title{
A Systematic Review of Bilateral Upper Limb Training Devices for Poststroke Rehabilitation
}

\author{
A. (Lex) E. Q. van Delden, ${ }^{1}$ C. (Lieke) E. Peper, ${ }^{1}$ Gert Kwakkel, ${ }^{1,2}$ and Peter J. Beek ${ }^{1}$ \\ ${ }^{1}$ Research Institute MOVE, Faculty of Human Movement Sciences, VU University Amsterdam, Van der Boechorststraat 9 , \\ 1081 BT Amsterdam, The Netherlands \\ ${ }^{2}$ Research Institute MOVE, Department of Rehabilitation Medicine, VU University Medical Center, De Boelelaan 1117, \\ 1081 HV Amsterdam, The Netherlands
}

Correspondence should be addressed to A. (Lex) E. Q. van Delden, l.van.delden@vu.nl

Received 20 July 2012; Accepted 8 October 2012

Academic Editor: Stefano Paolucci

Copyright (C) 2012 A. (Lex) E. Q. van Delden et al. This is an open access article distributed under the Creative Commons Attribution License, which permits unrestricted use, distribution, and reproduction in any medium, provided the original work is properly cited.

\begin{abstract}
Introduction. In stroke rehabilitation, bilateral upper limb training is gaining ground. As a result, a growing number of mechanical and robotic bilateral upper limb training devices have been proposed. Objective. To provide an overview and qualitative evaluation of the clinical applicability of bilateral upper limb training devices. Methods. Potentially relevant literature was searched in the PubMed, Web of Science, and Google Scholar databases from 1990 onwards. Devices were categorized as mechanical or robotic (according to the PubMed MeSH term of robotics). Results. In total, 6 mechanical and 14 robotic bilateral upper limb training devices were evaluated in terms of mechanical and electromechanical characteristics, supported movement patterns, targeted part and active involvement of the upper limb, training protocols, outcomes of clinical trials, and commercial availability. Conclusion. Initial clinical results are not yet of such caliber that the devices in question and the concepts on which they are based are firmly established. However, the clinical outcomes do not rule out the possibility that the concept of bilateral training and the accompanied devices may provide a useful extension of currently available forms of therapy. To actually demonstrate their (surplus) value, more research with adequate experimental, dose-matched designs, and sufficient statistical power are required.
\end{abstract}

\section{Introduction}

As technology advances, a growing number of mechanical and robotic training devices (i.e., mechanical devices with electronic, computerized control systems) for upper limb training have been proposed for stroke rehabilitation. Compared to conventional therapies, these training devices have the advantage that they allow a self-controlled increase in training intensity and frequency as well as the opportunity to train independently [1-4]. In recent years, a substantial number of these training devices have been designed specifically for bilateral upper limb training, but an integral overview and evaluation have thus far been lacking. The present study seeks to fill this lacuna.

Bilateral upper limb training is by no means a new form of stroke rehabilitation. Since days long past, therapists have been creative in using appliances, such as pulleys, to move the most impaired upper limb simultaneously with the less impaired upper limb [5]. Nevertheless, the current upsurge in the interest in bilateral upper limb training has a relatively short history and arose partly serendipitously $[6,7]$ and partly from insights gleaned from the motor control literature. In this literature, coupling (or interaction) effects between the two upper limbs have been investigated extensively in rhythmic interlimb-coordination studies involving healthy subjects [8-12]. It is well established that healthy subjects show a basic tendency towards inphase (i.e., symmetrical movements) or anti-phase (i.e., alternating movements) coordination, with a prevalent $1: 1$ frequencylocking mode for upper limb bilateral movements [12]. These tendencies reflect the coupling between the upper limbs. Based on the assumption that this coupling facilitates the functional recovery of the paretic arm, it is exploited in bilateral upper limb training, usually by moving both arms 
and/or hands in either in-phase or anti-phase coordination. However, whether one pattern is to be preferred over the other is currently not evident.

Recent systematic reviews produced mixed results on the superiority or inferiority of bilateral upper limb training over other interventions in poststroke rehabilitation. Two such reviews found strong evidence in support of bilateral upper limb training after stroke $[13,14]$. Another review was more reticent in its conclusions than the previous two [15], and three systematic reviews concluded that bilateral training is at best similarly effective as other treatments but certainly not better [16-18]. These mixed results may be related to the heterogeneity of types of bilateral upper limb training and the devices used in clinical trials. Therefore, an overview and evaluation of clinical applicability of bilateral upper limb training devices may be helpful in directing future research in this regard.

The present systematic review evaluates bilateral training devices designed for poststroke upper limb training in terms of (1) mechanical and electromechanical characteristics, (2) supported movement patterns, (3) targeted part and active involvement of the upper limb, (4) training protocols, (5) outcomes of clinical trials, and (6) commercial availability. The aim of the paper is to evaluate these aspects in a qualitative manner because not sufficient randomized clinical trials are available on the devices in question to evaluate or compare clinical outcomes statistically. We therefore aim at comparing and integrating concepts, findings, and insights from largely qualitative studies, culminating in an overview of training devices for poststroke rehabilitation, an assessment of their clinical applicability, and some general conclusions and recommendations for future developments and research.

\section{Methods}

Bilateral upper limb training device was defined as a device developed for upper limb rehabilitation that is either specifically designed for bilateral training or at least supports bilateral training as one of the prominent training modes, where both upper limbs perform simultaneous movements with one limb moving actively and the other limb moving actively, passively, or with assistance.

Relevant literature was identified through computerized and manual searches in the following electronic databases: PubMed, Web of Science, and Google Scholar. These databases were searched using the following MeSH headings and key words:

(i) cerebrovascular disorder $\$$, cerebrovascular accident, CVA, stroke, hemiparetic stroke, paresis, hemiparesis, hemiplegia,

(ii) upper extremit\$, upper limb\$, arm\$, forearm\$, wrist $\$$, hand $\$$, finger\$,

(iii) bilat\$, bimanual\$

(iv) robot $\$$, mechan $\$$, device.

Bibliographies of review articles, empirical articles, and abstracts published in conference proceedings were also examined. In further iterations, references from retrieved articles were examined to identify additional relevant articles. In light of the recent interest in bilateral upper limb training in stroke rehabilitation literature $[6,19]$, only articles published from 1990 onwards were searched.

Devices were selected for discussion if they met with the aforementioned definition of a device for bilateral upper limb training. Devices were categorized as robotic devices when they met the description of the PubMed MeSH term of robotics. All other devices were categorized as mechanical devices. Both categories are discussed in separate paragraphs.

\section{Results}

The search resulted in 311 single citations of which 70 reported on 20 different bilateral training devices. Of these, 6 were mechanical and 14 were robotic devices.

\subsection{Mechanical Devices}

3.1.1. BATRAC (Tailwind). Bilateral arm training with rhythmic auditory cueing (BATRAC) was introduced in 2000 [20], together with a custom-made bilateral arm trainer. The device consists of two independent T-bar handles mounted on nearly frictionless tracks that can move in the transverse plane perpendicular to the user. The handles have to be pushed forward and pulled back, either with both upper limbs simultaneously (in-phase) or alternatingly (antiphase), at a frequency paced by a metronome providing auditory cues. If a patient is unable to hold the handle with the hand of the most impaired upper limb, the hand is strapped onto it. The original BATRAC protocol focuses expressly on shoulder and elbow function. A modified version of the original BATRAC protocol focuses on distal upper limb function [21]. For the purpose of the latter protocol, a device was developed consisting of two manipulanda with handgrip that can be mounted on the distal ends of a chair's arm rests (see Figure 1(a)). The manipulanda allow flexion and extension movements of the wrist in the horizontal plane and have to be moved rhythmically in pace with an auditory metronome in either a mirror-symmetrical (in-phase) or an alternating (anti-phase) fashion. Visual feedback is provided in the form of a Lissajous display, left and right hand movement amplitudes, and the relative phase (and its variability) between both hands (see Figure 1(b)).

The original BATRAC protocol was first used in a pilot effect study involving a single group of patients with chronic stroke (i.e., more than 6 months after stroke onset) [20]. Fourteen patients received 6 weeks of BATRAC, three times a week, four times 5 minutes per session. Posttreatment assessment revealed improvements in Fugl-Meyer Assessment (FMA), Wolf Motor Function Test (WMFT), University of Maryland Arm Questionnaire for Stroke measuring daily use of the most impaired upper limb, as well as strength measures and range of motion measures for the most and less impaired upper limb. Most of these benefits sustained at 8-week followup.

Another study [22] examined the efficacy of a modified version of the BATRAC protocol. In this study, 


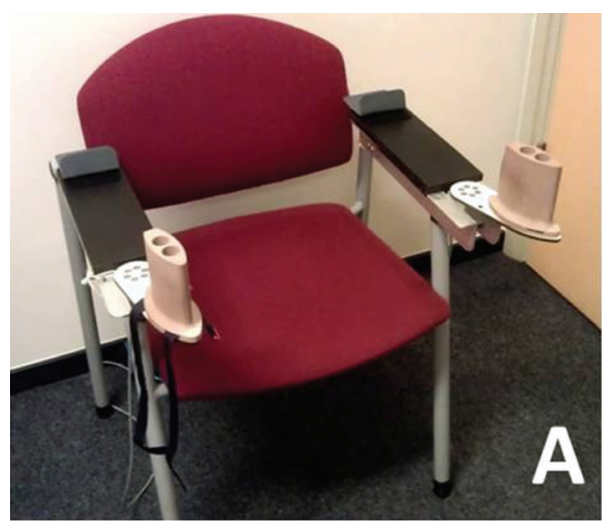

(a)

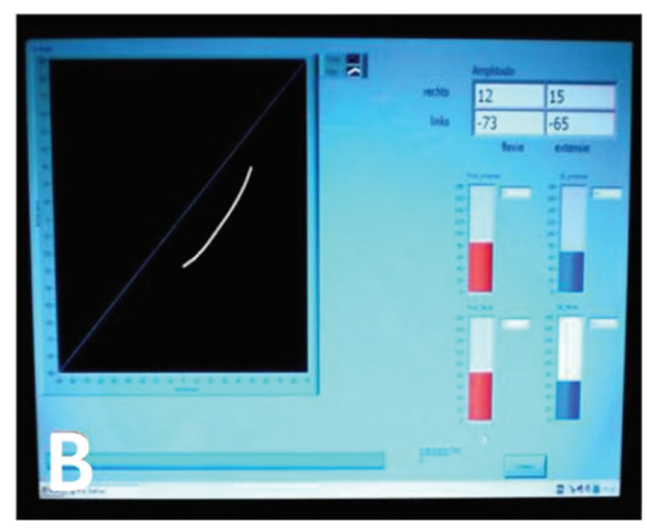

(b)

FIgURE 1: Modified BATRAC apparatus. (a) Manipulanda with handgrips. (b) Feedback, with Lissajous plane on the left (movements of left hand plotted against those of the right hand) and feedback regarding amplitudes and relative phase (and its variability) on the right. Reprinted with permission.

14 patients with chronic stroke participated in four 2-hourplus BATRAC sessions per week for 2 weeks. Although no significant changes in FMA or WMFT were found as a result of this intervention, patients reported an increase in daily use of the most impaired upper limb as evidenced by a significant change on the Motor Activity Log (MAL).

In a large RCT with patients with chronic stroke, BATRAC was compared with dose-matched therapeutic exercises [23]. A total of 111 patients were randomized over both intervention groups and received 6 weeks long 3 training sessions per week. The improvements in upper limb function were comparable between both groups posttreatment and retained after 4 months. There were however greater adaptations in brain activation after BATRAC than after the control treatment, suggesting that both treatments referred to different underlying neural mechanisms.

Currently, an RCT with 60 patients in the subacute phase after stroke (1-6 months) is being conducted [21]. In this RCT the BATRAC device for distal upper limb movements (Figure 1) is used and compared to constrained-induced movement therapy and conventional physical therapy. This RCT will be completed by the end of this year and will be reported in 2013/2014.

A commercial version of BATRAC, called Tailwind (see Figure 2), is produced and sold by Encore Path, Inc. Baltimore, MD, USA and is also available at Anatomical Concepts UK Ltd., Clydeland, Scotland. This device is produced for home-based training and differs from the original device, as the Tailwind also allows upward and outward movements. The BATRAC device for movements about the wrist is not commercially available.

3.1.2. Reha-Slide Duo and Reha-Slide (Nudelholz). The RehaSlide Duo (see Figure 3) consists of a board with two sledges running on parallel tracks [24]. Two handles on the sledges can be moved forward and backward separately, similar to the Tailwind used for BATRAC. The board on which the tracks are placed can be inclined up to $20^{\circ}$ for

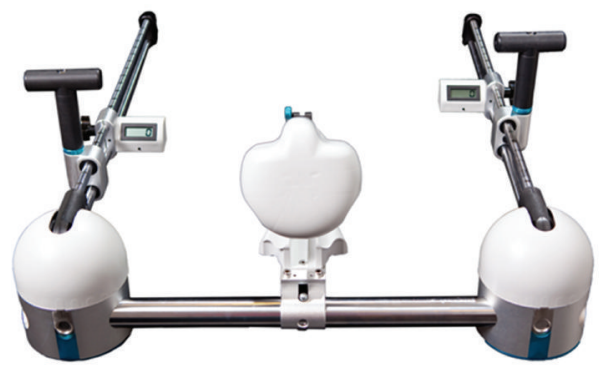

Figure 2: Tailwind. Reprinted with permission (http://www.tailwindtherapy.com/).

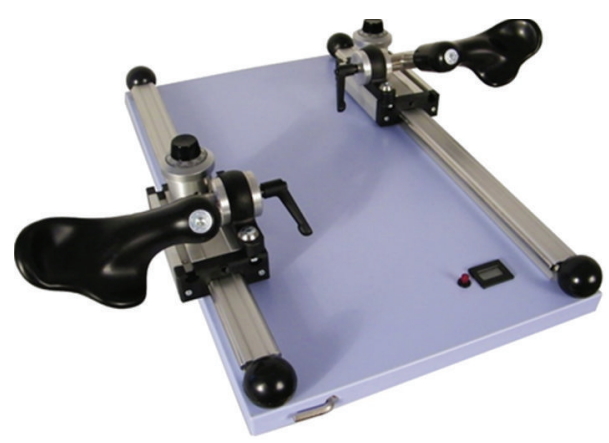

FIgure 3: Reha-Slide Duo. Reprinted with permission (http://www .reha-stim.de/).

upward movements, and friction for forward and backward movements can be adjusted for both handles separately via adjustable rubber brake elements in a range from $5 \mathrm{~N}$ to $80 \mathrm{~N}$ (with the board horizontal).

The Reha-Slide Duo was incorporated in an arm-studio for upper limb rehabilitation [24]. However, no clinical results have been reported to date specifically for the RehaSlide Duo. 


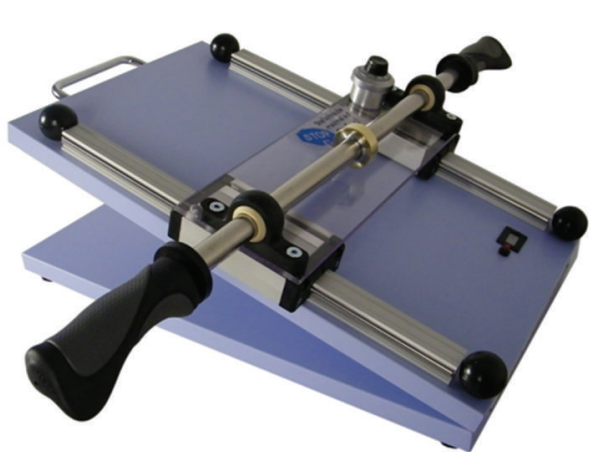

Figure 4: Reha-Slide (Nudelholz). Reprinted with permission (http://www.reha-stim.de/).

The Reha-Slide (Nudelholz, see Figure 4) consists of the same inclinable board with parallel tracks and sledges as the Reha-Slide Duo; however, an additional rod connects the two handles $75 \mathrm{~cm}$ apart from each other on either side, similar to a rolling pin [25]. The rod can be moved forward and backward by $30 \mathrm{~cm}$ (elbow extension and flexion), sideways in both directions by $15 \mathrm{~cm}$ (shoulder abduction and adduction), and it can be rotated by $360^{\circ}$ (wrist flexion and extension). The handles are yoked, so that the less impaired upper limb can drive the most impaired upper limb during training. The board on which the tracks are placed can be inclined up to $20^{\circ}$ for upward movements, and friction for forward and backward movements can be adjusted via adjustable rubber brake elements in a range from $5 \mathrm{~N}$ to $80 \mathrm{~N}$ (with the board horizontal). A wireless computer mouse can be centrally fixed to the rod for interaction with computer software (games and biofeedback).

The Reha-Slide was first tested with 2 patients 5 and 6 weeks after stroke [25]. Both patients trained for 20-30 minutes every workday for 6 weeks using the Reha-Slide in addition to a 10 week in-patient rehabilitation program of 4 times per week 45 minutes of physiotherapy and 3 times per week 45 minutes of occupational therapy following principles of neurodevelopmental therapy (NDT). RehaSlide treatment included training of the forward-backward movement cycles and drawing a square clockwise and counter-clockwise, while rotating the wrists. These exercises were repeated with the board inclined such that the patient moved his or her hands to shoulder level. The total number of movement cycles practiced was about 400 . Both patients showed improvements in muscle strength and FMA scores.

In an RCT the effects of treatment with the RehaSlide were compared with electrical stimulation of wrist extensors [26]. Fifty-four in-patients 4-8 weeks after stroke were randomized over two groups. The Reha-Slide group received the same protocol as described above [25]; however, the degree of inclination was always set in such a way that the handles reached the shoulder level, and forwardbackward friction level was increased over the weeks of the training. A metronome helped to pace movements, and at the end of each session, patients played a computer game of their choice for 5 minutes. The electrical stimulation group practiced 60-80 wrist extension repetitions per 30-minute

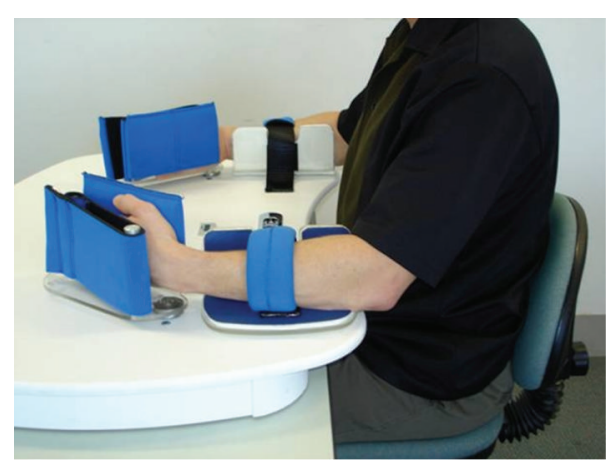

FIGURE 5: The Rocker for APBT. Reprinted with permission.

session. All patients also participated in an 8-10 week inpatient rehabilitation program based on NDT principles, including 5 45-minute sessions of physiotherapy and 4 30minute sessions of occupational therapy per week. FMA score and muscle strength improvement did not differ significantly between groups, both after treatment and at 3-month followup. Posttreatment improvement in the Box and Block Test (BBT) was greater in the Reha-Slide group, but this difference disappeared at 3-month followup. Muscle tone (measured with the MAS) differed significantly over the groups at 3-month followup, with an increase in tone in the electrical stimulation group.

The Reha-Slide and Reha-Slide Duo are commercially available at Reha-Stim, Berlin, Germany.

3.1.3. APBT (the Rocker). The device used for active-passive bimanual movement therapy (APBT, see Figure 5) is a custom-built system of connected crankshafts located in the body of a unit that couples two manipulanda [27]. It supports mirror symmetrical or near-symmetrical (a phase lag of $60^{\circ}$ ) coordination of wrist flexion and extension movements in the horizontal plane. With this system an actively moving less impaired upper limb can passively move the most impaired upper limb in either a synchronous or $\left(60^{\circ}\right.$ phase lag) asynchronous fashion.

For a pilot study, 9 patients with stroke (onsets ranging from 2-84 months before recruitment) participated in six 10-minute sessions of APBT per day, for 4 weeks [27]. The intervention was rhythmically practiced at a selfpaced movement rate (approximately $1.2 \mathrm{~Hz}$ ). Five patients practiced synchronous movements and four patients practiced asynchronous movements. Patients were instructed to maintain their most impaired upper limb muscles at rest. After treatment, five of the nine patients (from both groups) had increased their FMA scores by more than 10\%. These improvers also showed a decrease in affected cortical map volume. There was no follow-up assessment.

In an RCT, 32 patients with chronic stroke were randomized over two groups [28]. Patients in the control group received a set of wooden blocks and were instructed to perform two self-directed, home-based tasks (transporting and manipulating the wooden blocks) with their most impaired upper limb for 10 minutes, 3 times per day, for 
4 weeks. Patients in the APBT group received the same set of wooden blocks and the same instructions, but had also access to the APBT device. They were instructed to use the APBT device for mirror-symmetric movements of the wrists for 1015 minutes prior to the exercises with the blocks. Attention first had to be focused on the less impaired upper limb at the beginning of each session, followed by a gradual shift towards the most impaired upper limb. In the third week, they were instructed to imagine they were actively producing the movements of their most impaired wrist, with progression towards bilaterally active movements in the fourth week. At 1-month follow-up FMA change scores were significantly greater in the APBT group than in the control group. This difference was not present at the immediate posttreatment assessment. There were no significant differences in grip strength and National Institutes of Health Stroke Scale scores at any posttreatment assessment. The APBT group exhibited significant increases in ipsilesional M1 excitability, transcallosal inhibition from ipsilesional to contralesional M1, and intracortical inhibition within contralesional M1, both immediately posttreatment and at 1-month follow-up. These changes were absent in the control group at both assessment times. Note that the interventions were not dosematched: the APBT group spent more time training.

The effects of additional APBT were also assessed in a single case series study with two matched pairs in the subacute phase after stroke [29]. In addition to usual standard care treatment consisting of daily occupational therapy and physical therapy, all patients received 20-30 minutes of motor practice 1 or 2 times per day, 5 days per week, for 1-3 weeks. Motor practice included tasks that were designed to improve joint stability, mobility, and strength, as well as reaching, and grasp and release exercises. On top of that, patients receiving additional APBT practiced mirror-symmetrical wrist flexion and extension movements for 10 minutes prior to motor practice. The magnitudes of improvements on the FMA and Action Research Arm Test (ARAT) at posttreatment as well as 1-month follow-up assessments were greater in patients who received additional APBT. Again the interventions were not dose-matched.

The APBT device (the Rocker) is not commercially available.

3.1.4. Able-X. Recently, a movement-based game controller (the CyWee Z) has been incorporated into a custom-built handlebar (Able-X, see Figure 6) in order to render it suitable for bilateral use [30]. With the CyWee $\mathrm{Z}$ positioned in the handlebar, rotations in the transverse plane produce horizontal mouse cursor translations on a computer screen, while rotations in the sagittal plane produce vertical mouse cursor translations. Using a suite of games a graduated series of physical challenges can be provided with the CyWee Z.

Fourteen patients with chronic stroke participated in a study aimed to determine the effectiveness of a bilateral, self-supported upper limb rehabilitation intervention using the handlebar with CyWee Z [30]. All patients received a control treatment, followed by a washout period, and finally the intervention. During the initial control period, patients played four simple mouse-based computer games

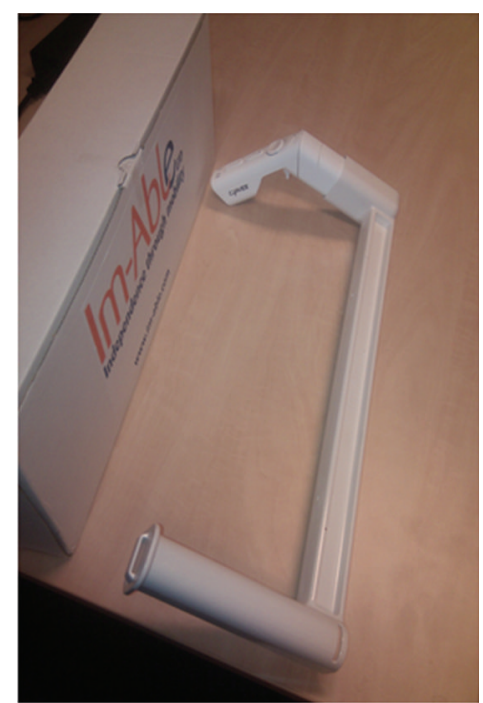

Figure 6: Able-X with CyWee Z. Reprinted with permission.

on a personal computer (PC) with their less impaired upper limb. The training was provided in group sessions, with three participants playing on three PCs under supervision of one or two therapists, during a 2.5 week period encompassing 8 to 10 sessions, each lasting between 45 and 60 minutes. Then followed a period of 2-3 weeks during which no intervention was provided. Finally, patients received the intervention comprised of playing games on a PC using the custom-built CyWee Z handlebar. As in the control treatment, three participants played on three PCs supervised by one or two therapists, that is, the same amount of therapist interaction was provided for the two types of treatment. The intervention lasted for 8-10 sessions of 45-60 minutes over a period of 2.5 weeks. During each session, the participants made an estimated minimum of 500 repetitions and possibly up to 800 repetitions. One repetition could be an up-down movement, a left-right movement, or (in most cases) a combination of both (e.g., moving cursor from center to top-right corner of screen). Scores on the FMA improved significantly after intervention. Scores on the WMFT and Disabilities of Arm, Shoulder and Hand (DASH) did not improve.

The Able-X handlebar (including CyWee Z) is commercially available at http://www.im-able.com/.

\subsection{Robotic Devices}

3.2.1. $\mathrm{H}-\mathrm{O}-\mathrm{H}$. The Hand-Object-Hand (H-O-H) system (see Figure 7) was one of the first bilateral training devices to be introduced in stroke rehabilitation [19]. This particular robotic training device was presented as an introduction to the design of more complex robotic assistance devices for poststroke upper limb rehabilitation. In the $\mathrm{H}-\mathrm{O}-\mathrm{H}$ system both hands are placed in two rigid handles that constrain the patient's movements to flexions and extensions about the wrists, that is, to a single degree of freedom (DOF) per hand, which have to be coordinated. A $17.5 \mathrm{~cm}$ long 


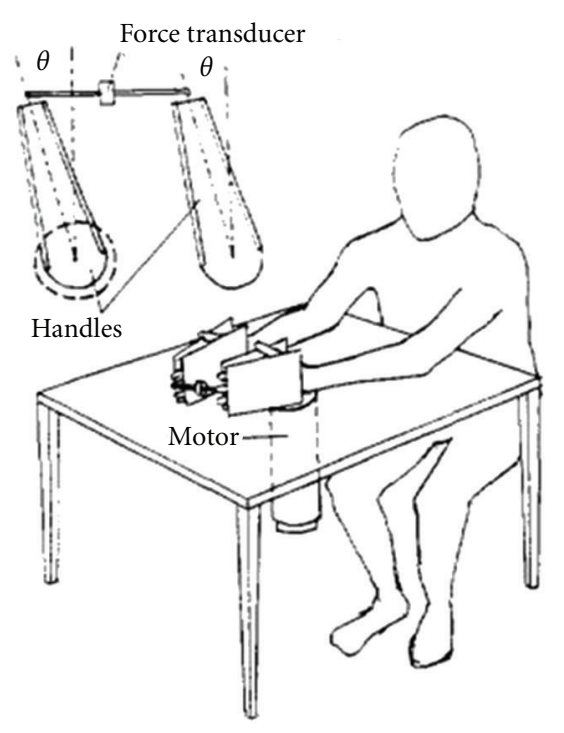

Figure 7: H-O-H. Inset: top-view of the force transducer between the distal parts of the handles. Reprinted with permission.

pencil-like object consisting of two machine screws attached to both sides of a force transducer is placed between the distal parts of the handles. The robotic aid consists of a computercontrolled motor mounted underneath one of the handles. The system is capable of partially or fully substituting for one hand in two tasks: rhythmically moving the object from left to right and vice versa (i.e., parallel movements for the hands) and squeezing.

Even though the $\mathrm{H}-\mathrm{O}-\mathrm{H}$ system has been advocated for poststroke upper limb training, no clinical results have been reported to date, nor is the system commercially available.

3.2.2. The Bimanual Lifting Rehabilitator. The Bimanual Lifting Rehabilitator (see Figure 8) is a 2-DOFs linkage that captures the dynamics of bimanual lifting [31]. One of the two links consists of the object to be lifted with two handles, while the other link provides both measurement of the object's vertical position and assistance when the force of one arm is inadequate or insufficient. The computer-controlled motor, positioned near the elbow and attached to the link alongside the forearm of the most impaired side, adjusts the lifting force of the most impaired upper limb by applying torque to the controlled link when the link to be lifted is tilted. Tilt is measured with a potentiometer positioned near the bearing between the two links. The Bimanual Lifting Rehabilitator is capable of partially or fully substituting the force of one upper limb, for example when lifting a cafeteria tray carrying a cup of coffee.

Like the H-O-H system, no reports of clinical tests of the Bimanual Lifting Rehabilitator are available, nor is the device commercially available.

3.2.3. MIME. The Mirror Image Movement Enhancer (MIME, [32]) is a 6-DOFs robot manipulator (PUMA 560, Stäubli Inc.), equipped with actuators to apply forces in goal-directed movements (see Figure 9). The MIME system

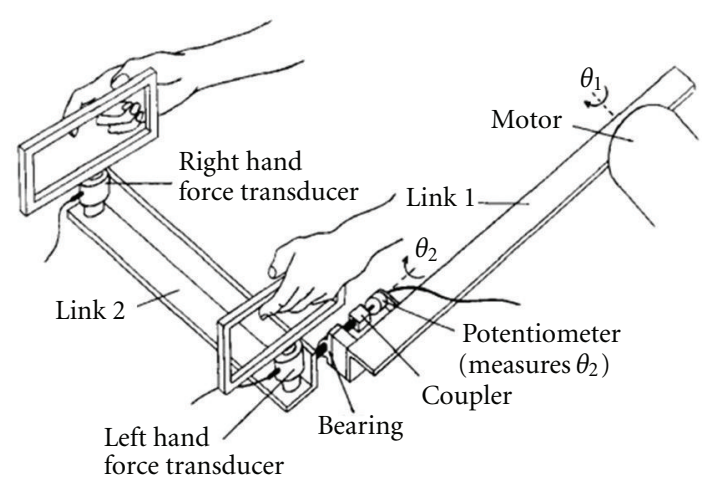

FIgURE 8: The Bimanual Lifting Rehabilitator. Reprinted with permission.

focuses on shoulder and elbow function. The most affected forearm and hand are strapped in a customized splint, which restricts wrist and hand movement and is attached to the end effector. The less affected upper limb is strapped in a similar splint attached to a position digitizer. The robot's 6 DOFs allow the forearm to be positioned in a large range of positions enabling the patient to practice quite complex 3D movement patterns. A 6-axis sensor measures the forces and torque between the most affected upper limb and the robot. MIME supports four modes of robot-assisted movement. In the bimanual mode the patient is instructed to perform bimanual mirror-image movements while the robot assists the most affected upper limb by continuously moving the most affected upper limb to the contralateral forearm's mirror-image orientation and position. The passive, activeassisted, and active-resisted modes are unilateral training modes.

The MIME system has been used in three randomized clinical trials (RCTs). In the first RCT [33], the effects of the MIME system were compared with NDT targeting the upper limb in patients with chronic stroke. In total 27 patients received 24 1-hour sessions of therapy over 2 months. A therapist blinded to group assignment evaluated outcomes on the FMA, Functional Independence Measure (FIM), and biomechanical measures of strength and reaching kinematics. After 2 months of training the group receiving treatment with the MIME system had larger improvements in the proximal movement portion of the FMA, larger gains in proximal strength, and larger increases in reach extent than the group receiving NDT. The FMA difference between groups disappeared at 6-month followup. However, the MIME system group showed larger improvements in the FIM at 6-month followup; a difference that was not present directly after treatment. In this RCT all four modes of robot-assisted movement were used, which means that the group receiving treatment with the MIME system spent approximately 12 minutes of each session in the bilateral mode and 25 minutes in unilateral modes. Hence, it is unclear which type of training (bilateral or unilateral) contributed most to the observed improvements.

In a second RCT [34], patients with stroke in the subacute phase were recruited and divided over four groups. 


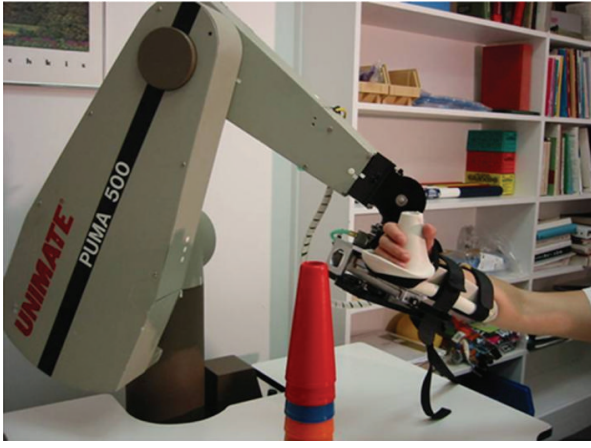

(a)

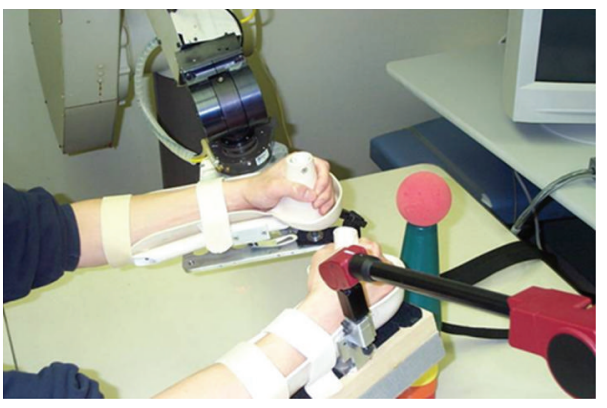

(b)

Figure 9: MIME. (a) Unilateral mode. (b) Bilateral mode. Reprinted with permission (funding: US Department of Veterans Affairs Rehabilitation Research and Development Program).

One group received NDT targeting the proximal upper limb $(n=6)$ and three groups received robot-assisted treatment: bilateral $(n=5)$, unilateral $(n=9)$ or combined unilateral and bilateral $(n=10)$. All groups received 15 1-hour treatment sessions over 4 weeks. Compared to the NDT group, only the combined unilateral and bilateral (robot-assisted) group showed greater gains in the proximal FMA and the synergy scale of the Motor Status Score after treatment. However, this difference disappeared after 6 months. At the 6-month followup all gains in all treatment groups were equivalent, except that the unilateral group showed greater improvement in the distal FMA than the combined unilateral and bilateral group.

Results of a third RCT, involving patients between 7 to 21 days after stroke, were reported last year [35]. Fiftyfour patients were randomized over three groups receiving treatment for 3 weeks. The low-dose robot-assisted group received up to 15 hours and the high-dose robot-assisted group up to 30 hours of training with the MIME system in all four modes. The third group received up to 15 hours of conventional upper limb training. After treatment the high-dose robot-assisted group showed larger gains in FIM scores than the conventional training group. At the 6-month followup this difference was no longer present. There was however a significant difference between the high-dose and low-dose robot-assisted groups on the Modified Ashworth Scale (MAS), with the former having larger increase in

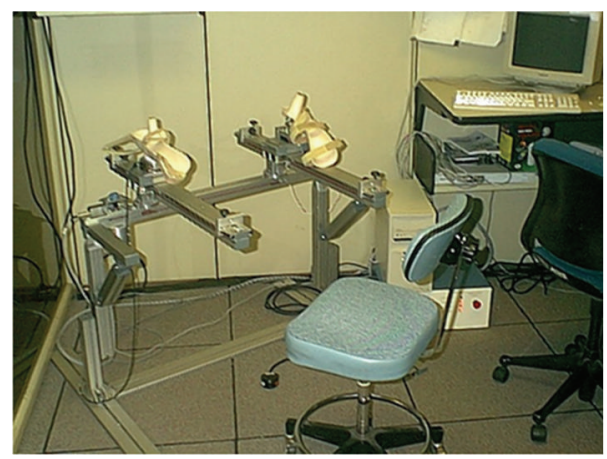

Figure 10: ARCMIME. Reprinted with permission (funding: US Department of Veterans Affairs Rehabilitation Research and Development Program and National Institutes of Health Phase I Small Business Innovative Research).

muscle tone than the latter; a difference that was not present immediately after treatment.

The MIME system is not commercially available.

3.2.4. ARCMIME. The ARCMIME (see Figure 10) was designed in an attempt to develop a clinically viable, commercial device with similar clinical outcomes as the MIME system [36]. The ARCMIME structure consists of aluminum extrusions and linear slides on which arm splints are mounted. The system was designed such that it could be manually adjusted and reconfigured. The pitch angle can be adjusted $85^{\circ}$ from horizontal and the two arms with the linear slides can be rotated $345^{\circ}$ around their individual pivot points. Unlike the MIME system, which allows movements in $3 \mathrm{D}$, the ARCMIME only allows movements along the linear slides.

Four patients with stroke and two healthy subjects performed tasks with the ARCMIME as well as the MIME system to compare the operation of both systems [36]. Ten trials of each of the four modes were performed at each of two movement trajectories. When movement patterns were matched, the force directed towards the targets by the paretic limb were not significantly different for the two systems. From these results it was concluded that the clinical gains seen with the MIME system may be reproducible with the ARCMIME system.

The ARCMIME system is not commercially available.

3.2.5. Braccio Di Ferro. The Braccio di Ferro (see Figure 11) is a planar manipulandum with 2 DOFs operated by two motors [37]. The operational plane can be changed from horizontal to vertical. The Braccio di Ferro can generate four types of forces: an assistive field, a resistive elastic field, a rigid "wall," and a viscous field. This haptic robot was designed primarily for unilateral use. However, by substituting the handle with a length-adjustable bar that can rotate freely, the Braccio di Ferro can also be used for bilateral training. Bar rotations are measured with a potentiometer.

Bilateral training with the Braccio di Ferro was tested with patients with chronic stroke. In two feasibility studies 


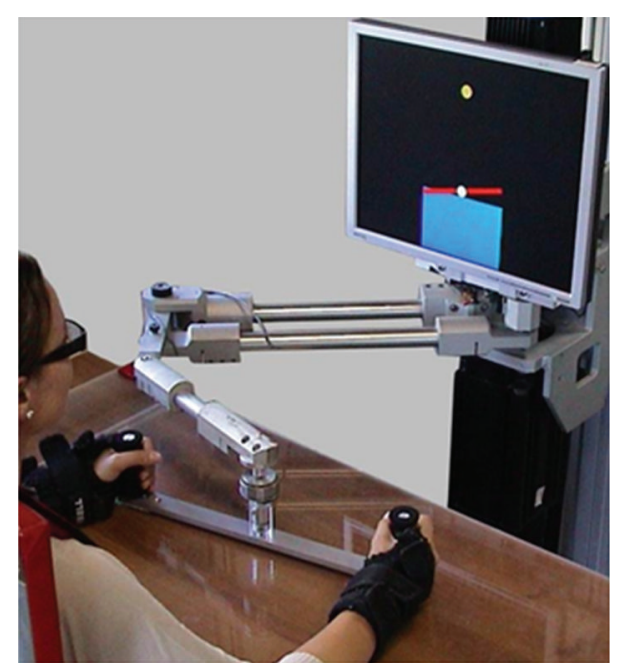

Figure 11: Braccio di Ferro. Reprinted with permission (http:// www.iit.it/).

$[38,39]$ patients performed forward and backward movements in the Braccio di Ferro generating the four types of forces described above. The training cycle consisted of five sessions over 2 to 3 weeks, each session lasting no more than 45 minutes. All patients improved their performance: movements became faster, smoother, more precise, and required decreasing levels of assistive force. However, no clinical outcomes have been reported for the device.

The Braccio di Ferro is not commercially available.

3.2.6. Bimanual Handlebar. The bimanual handlebar [40] has two active DOFs (see Figure 12) allowing forward and backward translational movements as well as rotational movements of the end-effector around an axis perpendicular to the direction of the translation. The bimanual handlebar is made of two parts, which are both mounted on the endeffector and independently measure forces generated by each upper limb using two 6-DOFs force and torque sensors. The complete handlebar turns like a steering wheel and can actively resist the subject's movements. An LCD screen is mounted on the top of the handlebar to enable visual representation of a virtual task. The subject's task is to track a reference object on the screen by moving the robot endeffector, which is also displayed on the screen with a tracker object. In the bilateral mode, the subject holds the handlebar with both hands and must coordinate them to keep the tracker object's orientation constant.

No clinical tests have been reported to date for this device and it is not commercially available.

3.2.7. Bi-Manu-Track. The research group that developed the Reha-Slide also developed the Bi-Manu-Track [41]. The Bi-Manu-Track is a computerized motor-driven arm trainer that allows bilateral training of two movement patterns: forearm pro- and supination and wrist flexion and extension (see Figure 13). To switch between movement patterns the device can be tilted by $90^{\circ}$. The Bi-Manu-Track supports

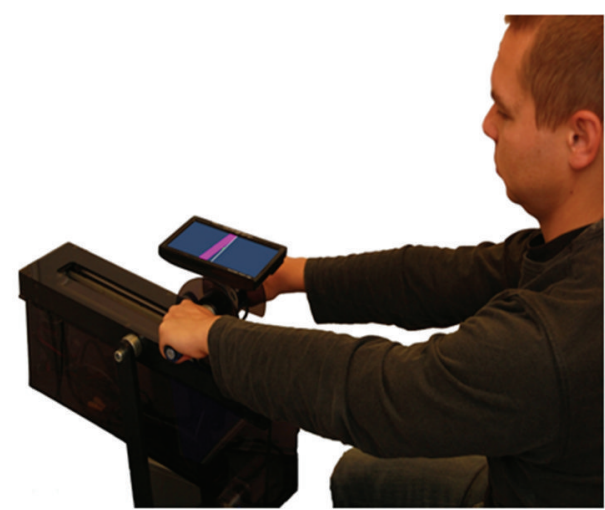

Figure 12: Bimanual handlebar. Reprinted with permission.

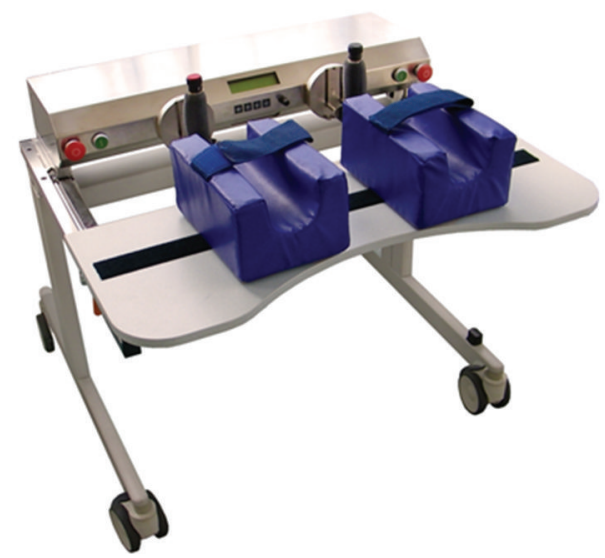

Figure 13: Bi-Manu-Track. Reprinted with permission (http:// www.reha-stim.de/).

three computer-controlled modes of practice. In the passivepassive mode the robot controls both arms. In the activepassive mode the less impaired upper limb actively moves the handle while the robot guides the most impaired upper limb. In the active-active mode both arms perform actively by overcoming an initial isometric resistance. Movements can be either mirror-symmetric (in-phase) or parallel (antiphase). Amplitude, speed, and resistances can be set individually.

The Bi-Manu-Track was tested first in 12 patients with chronic stroke [41]. On top of an ongoing comprehensive rehabilitation program patients received daily upper limb training of 15 minutes in all three modes of the Bi-ManuTrack on weekdays, for 3 weeks. After treatment MAS scores revealed significant muscle tone reduction in wrist and fingers. However, scores returned to pretreatment values at 3month followup. Other scores, such as the Rivermead Motor Assessment, did not change significantly.

In an RCT the effects of treatment with the Bi-ManuTrack were compared with electrical stimulation treatment of the wrist extensor in patients with severe hemiparesis [42]. In total 44 patients 4 to 8 weeks after stroke were randomized over both groups. All patients trained for 20 minutes every workday for 6 weeks. The Bi-Manu-Track 
group performed 800 repetitions per session: 200 wrist cycles in the passive-passive mode and 200 in the active-passive mode, 200 forearm cycles in the passive-passive mode and 200 in the active-passive mode. These patients were also allowed to practice 25 to 50 repetitions in the active-active mode. The electrical stimulation group practiced $60-80$ wrist extension repetitions per session. Upper limb muscle power and FMA scores increased significantly more in the BiManu-Track group than in the electrical stimulation group posttreatment and at 3-month followup.

In another RCT the Bi-Manu-Track was used to test the effect of bilateral robot-assisted training with transcranial direct current stimulation (tDCS) [43]. In this study, 96 patients with severe upper limb impairment 3-8 weeks after stroke were randomized over three groups. For 6 weeks, one group received anodal stimulation of the lesioned hemisphere and one group received cathodal stimulation of the contralesional hemisphere for 20 minutes. The third group received sham stimulation. During stimulation patients practiced 400 repetitions of two different bilateral movements with the Bi-Manu-Track. The passive-passive and active-passive modes were used during training. BiManu-Track training and tDCS were additional to an ongoing comprehensive rehabilitation program consisting of daily ergometer training, a daily 45 minutes of physiotherapy, and 4 times per week 30 minutes of occupational therapy. All patients improved on the FMA and BBT, but there were no differences between groups posttreatment and at 3-month followup.

A third RCT compared the effects of Bi-Manu-Track training with dose-matched active control therapy [44]. Two groups of 10 patients with chronic stroke each received 90105 minutes of training each workday for 4 weeks. The BiManu-Track group practiced 300-400 forearm repetitions in the passive-passive mode, 300-400 in the active-passive mode, 150-200 forearm repetitions, and 150-200 wrist repetitions in the active-active mode within one session. For patients with active forearm pro- and supination or active wrist flexion and extension a fourth mode (passive-active) was introduced with the most impaired upper limb actively executing the training cycles. A computer software program was added to the Bi-Manu-Track training so that patients received immediate visual feedback about the actions or force they exerted during training. Following robot-assisted training patients practiced various functional activities for 15 minutes. The control group received a dose-matched training protocol, including NDT, with emphasis on functional task training. The Bi-Manu-Track group significantly increased FMA scores, upper limb activity ratio (assessed with accelerometers), the amount and quality of upper limb use (assessed with the MAL), and bimanual ability (assessed with the ABILHAND questionnaire). There were no differences on the FIM and no follow-up assessment is reported.

In a pilot study, examining 21 patients in the chronic phase after stroke, unilateral and bilateral robot-assisted training with the Bi-Manu-Track were compared with each other and with standard upper limb rehabilitation [45]. The bilateral Bi-Manu-Track group received training similar to that described above [44]. The unilateral Bi-Manu-Track group received a modified protocol and only trained the most impaired upper limb. In the active-active mode, the most impaired upper limb had to move the handle against a resistance (set by a therapist) through the whole movement (in contrast to only overcoming an initial resistance). The control group received dose-matched treatment with activities involving weight bearing, stretching, strengthening, and unilateral and bilateral coordination, and fine motor tasks. The unilateral Bi-Manu-Track group improved more on the FMA overall score, proximal subscore, and distal muscle power than did the bilateral Bi-Manu-Track and control groups. However, the bilateral Bi-Manu-Track group had greater gains in proximal muscle power than the unilateral Bi-Manu-Track and control groups. There were no differences on the MAS and no follow-up assessment is reported.

The same research group carried out an RCT with patients with chronic stroke, comparing Bi-Manu-Track bilateral upper limb training with therapist-based bilateral upper limb training and a control treatment [46]. All groups received treatment for 90-105 minutes per session, 5 sessions per week, for 4 weeks. The bilateral Bi-ManuTrack group received training similar to that described above [44]. The therapist-based bilateral upper limb training group practiced a variety of bilateral functional tasks under oneon-one supervision of therapists. Treatment for the control group involved conventional therapeutic activities for the upper limb, unilateral and bilateral fine motor tasks, and compensatory practice on functional tasks. On the FMA, the therapist-based bilateral upper limb training group showed higher distal part scores than the control group. On the Stroke Impact Scale (SIS), the Bi-Manu-Track group showed better strength subscale, physical function domain, and total scores than the control group. In addition, kinematic variables differed between the three groups. The therapistbased bilateral upper limb training group demonstrated significantly better temporal efficiency and smoothness, straighter trunk motion, and less trunk compensation compared with the Bi-Manu-Track and control groups. The BiManu-Track group had increased shoulder flexion compared with the therapist-based bilateral upper limb training and control groups.

Currently, an RCT with patients in the chronic phase after stroke (6-24 months) is in operation (http://www .clinicaltrials.gov/, NCT01525979). In this 5-arms RCT treatment with the Bi-Manu-Track used in a unilateral and bilateral fashion is compared with unilateral, bilateral, or combined task-related upper limb training.

The Bi-Manu-Track is commercially available at RehaStim, Berlin, Germany.

3.2.8. Hand Robotic Rehabilitation Device. In 2009, Rashedi et al. [47] reported the design and development of another hand robotic rehabilitation device (see Figure 14). Like the Bi-Manu-Track, it enables bilateral training of two movement patterns: forearm pro- and supination and wrist flexion and extension movements. Three different modes were programmed for the device: a passive mode in which 


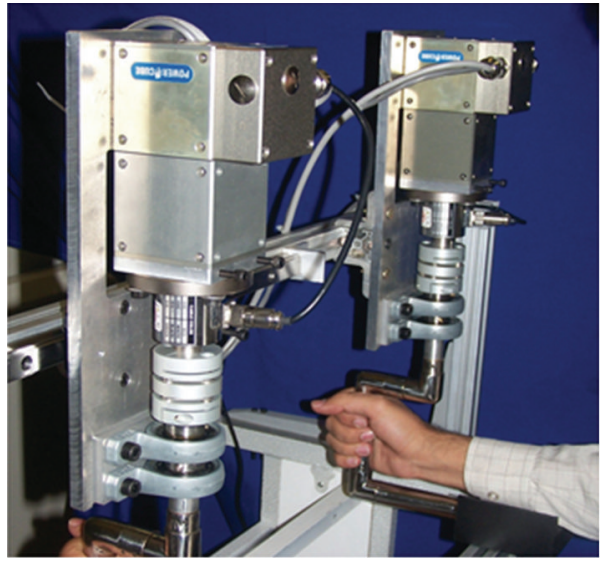

(a)

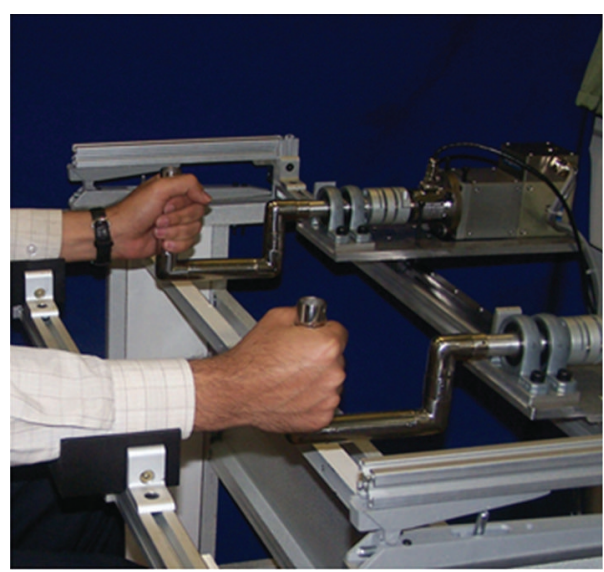

(b)

FIGURE 14: Hand robotic rehabilitation device. (a) Wrist flexion and extension. (b) Forearm pro-and supination. Reprinted with permission.

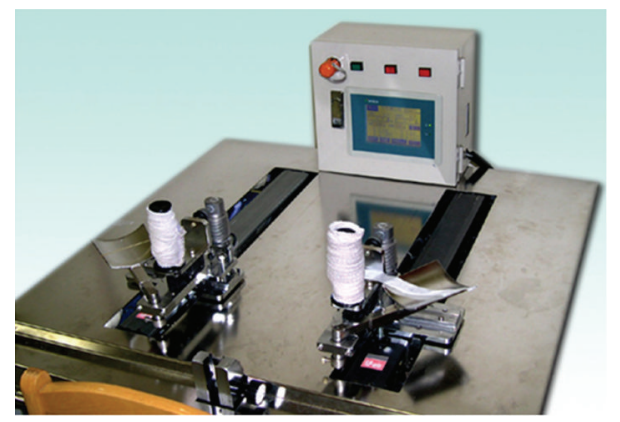

FIGURE 15: BFIAMT. Reprinted with permission (http://www.bmes .org.tw/).

the velocity and range of motion can be controlled separately, an active bimanual mode in which the less impaired upper limb moves the most impaired upper limb in a mirror-image motion pattern, and an active mode in which each side could move independently against an adjustable resistance.

No clinical tests have been reported for this device and it is not commercially available.

3.2.9. BFIAMT. The bilateral force-induced isokinetic arm movement trainer (BFIAMT, see Figure 15) is a robot-aided device with 2 servomotors, 2 parallel roller guides, 2 handles, 2 forearm troughs, 2 load cells, and a control panel [48]. The BFIAMT resembles the earlier described Reha-Slide (the handles can be moved forward and backward over parallel tracks), although with the BFIAMT the servomotors can provide aid or resistance during movement, and the load cells detect pull and push forces. The device supports four different treatment modes: bilateral passive, bilateral activepassive, bilateral reciprocal, and bilateral symmetric upper limb movement.

The BFIAMT was used in a single cohort study with patients with chronic stroke [48]. Patients followed a training program consisting of 40 minutes of training 3 sessions per week for 8 weeks. Treatment sessions consisted of two parts.
In Part 1 (30 minutes) patients completed 3 consecutive sets of 20 repetitions of bilateral symmetric arm push and pull movements with the BFIAMT. A subject's isometric maximal arm push and pull strength in both the less and most impaired upper limbs were identified before treatment. The preset demanded forces for the 3 sets were $10 \%, 20 \%$, and $10 \%$ of maximal push and pull forces of the affected and unaffected arms. In addition, subjects were instructed to perform bilateral symmetric push and pull movements at a comfortable cycling pace; the most preferred cycling frequency was $0.1 \mathrm{~Hz}$. During training bilateral push and pull forces were shown in real time to the patient as visual feedback. Generally, the subjects had to keep exerting the demanded forces bilaterally for 10 seconds to complete a smooth push and pull movement. In Part 2 of the treatment (10 minutes), patients received a conventional rehabilitation program focused on treatment that did not provide symmetric bilateral movement and resistance training to the upper limbs. This program included range of motion exercise, muscle tone normalization, compensatory activity of daily living training, postural control training, and gait correction. At posttreatment and 8-week follow-up assessments upper limb function (assessed with FMA, MAS, and Frenchay Arm Test) had improved significantly, as well as grip, push and pull strengths, and reaching kinematics.

The BFIAMT is not commercially available.

\subsubsection{Bimanual-Coordinated Training System. The} bimanual-coordinated training system is a master-slave system configured with two identical terminals, one for each limb [49]. Two length-adjustable handles for elbow flexion-extension movements are connected with the terminals and two torque transducers and a torque signal amplifier are applied to measure terminal torques and to verify the torque relationship between the two terminals. Furthermore, the system is configured with two identical motors and gearboxes. The working states of the two motors are determined by the forces exerted by the upper limbs on the two terminals. The terminal receiving the larger 


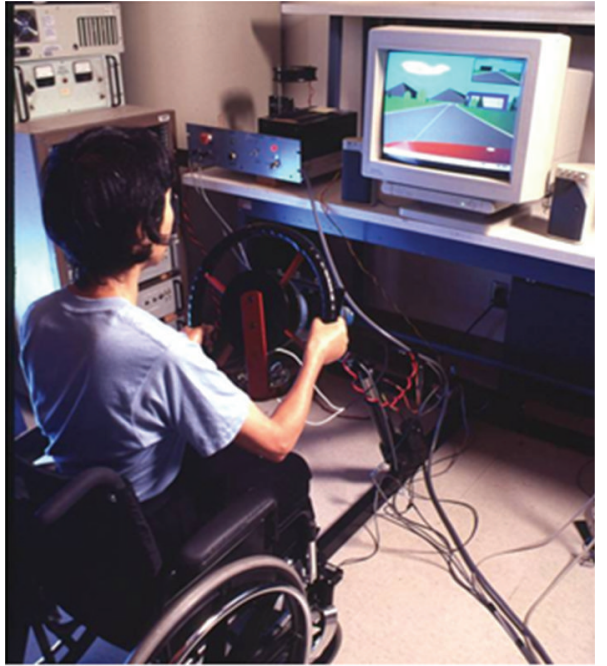

FIGURE 16: Driver's SEAT. Reprinted with permission (funding: US Department of Veterans Affairs Rehabilitation Research and Development Program).

force serves to generate state and behaves as the master terminal, while the other terminal works in electromotive state and behaves as the slave terminal. The system supports three training modes: (i) passive-driven mode with the less impaired upper limb driving the most impaired upper limb (which moves passively), (ii) active-assisted mode with the less impaired upper limb assisting the most impaired upper limb to complete desired movements, and (iii) activeresisted mode with the less impaired upper limb resisting the movement of the most impaired upper limb (here the motor operated by the most impaired upper limb behaves as the master, while the motor controlled by the less impaired upper limb behaves as the slave).

No clinical tests have been reported for this device and it is not commercially available.

3.2.11. Driver's SEAT. The Driver's Simulation Environment for Arm Therapy (Driver's SEAT; see Figure 16) was developed as a prototypical rehabilitation device [50]. Driver's SEAT is a 1-DOF robotic device with a servomotor and an adjustable-tilt split-steering wheel which measures position and force related performance. The split-steering wheel interfaces with driving simulator hardware that generates realistic graphical road scenes and collects data associated with steering dynamics. The steering wheel configuration measures the forces generated with each arm independently. The device allows three modes of operation: passive movement, active steering, and normal steering. In the passive movement mode the less impaired upper limb does the steering while the most impaired upper limb is moved passively with the help of the servomechanism. In the active steering mode subjects are instructed to steer with the most impaired upper limb while relaxing the contralateral upper limb. In this mode the servomechanism serves to encourage steering with the most impaired upper limb while involvement of the less impaired upper limb is actively discouraged through

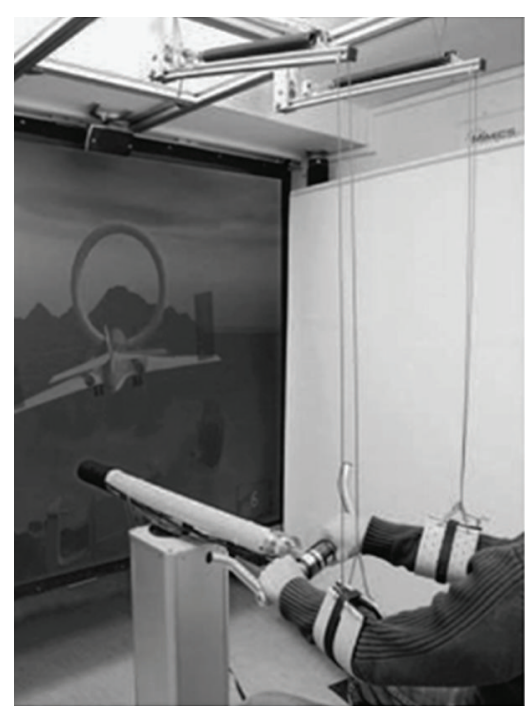

Figure 17: Adaptive Bimanual Robotic Training. Reprinted with permission.

a partial restraint. This restraint is invoked using corrective force cues defined as the stiffening of the wheel in proportion to the less impaired upper limb's tangential force on the wheel rim. In the normal steering mode subjects are encouraged to practice coordinated driving and improve their force symmetry by actively steering with both upper limbs.

In an experiment involving 8 patients with chronic stroke and 8 healthy subjects the effects of steering with (active steering mode) and without (normal steering) force cues were compared [51]. In patients with stroke, force cues significantly increased productive torque activity in steering directions up and against gravity with the most impaired upper limb.

The Driver's SEAT is not commercially available.

3.2.12. Adaptive Bimanual Robotic Training. Trlep et al. developed another robotic system designed for steering tasks [52]. This system uses the HapticMASTER robot system (FCS Control Systems, The Netherlands). The existing 3 DOFs of the HapticMASTER are expanded with an extra active joint at the end of the robot to allow the simulation of an active steering wheel. Bimanual handlebars mounted on the end-effector of the robot independently measure the forces generated by each arm. The handlebars turn like a steering wheel and can actively resist the subject's steering (see Figure 17). With both upper limbs supported by a passive gravity compensation mechanism, subjects can perform tasks in a virtual flight simulator environment. The robot is programmed to constrain the motion of the handlebars to the trajectory of three exercise modes: (i) vertical movement with active shoulder flexion with the elbow extended, (ii) horizontal movement with active elbow extension and shoulder protraction, and (iii) isolated active elbow extension. During exercise subjects are stimulated to use the most impaired upper limb against resistance produced by the robot. If the most impaired upper limb is 


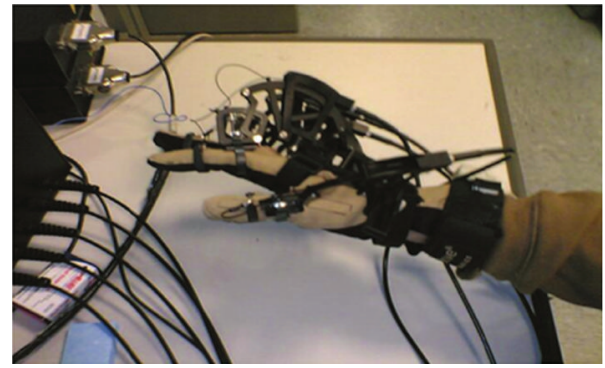

(a)

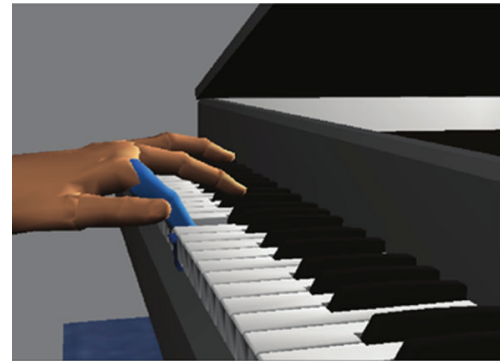

(b)

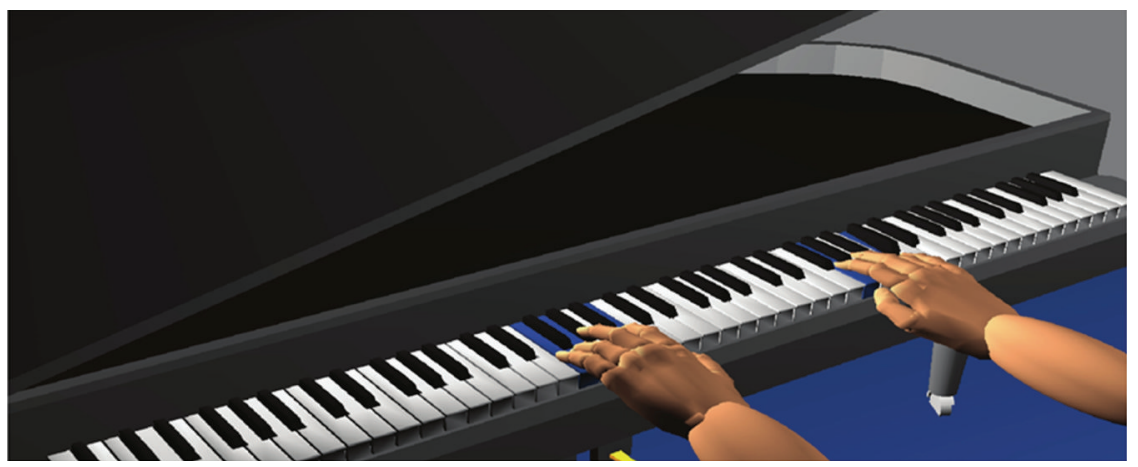

(c)

Figure 18: Virtual Reality Piano. (a) CyberGrasp worn over a CyberGlove. (b) A virtual key press. (c) Piano simulation with hands shown in a first-person perspective. Reprinted with permission.

not able to perform as required, the forces applied by the less impaired upper limb are scaled down using an adaptive gain to stimulate use of the most impaired upper limb.

The adaptive bimanual robotic training system was tested in 4 patients with chronic stroke [52]. All patients received 8 training sessions; 2 training sessions a week for a period of 4 weeks. Each training session consisted of the three exercises described earlier. Each exercise was first performed unilaterally using the less impaired upper limb, subsequently in the bilateral mode and finally as a unilateral exercise of the most impaired upper limb. Ten stimulated movements were performed in each training mode. Training of the three exercise modes resulted in improvements of task performance in bilateral and unilateral tasks of orientation and position tracking. No clinical test results were reported.

The adaptive bimanual robotic training system is not commercially available.

3.2.13. Virtual Reality Piano. The Virtual Reality Piano (see Figure 18) is a robotic/virtual environment system designed for upper limb training in patients with stroke [53]. The Virtual Reality Piano presents visual, auditory, and tactile feedback comparable to an actual piano by using a force reflecting exoskeleton (CyberGrasp). Upper limb tracking (with the CyberGlove) allows patients to train both the arm and hand as a coordinated unit. The Virtual Reality Piano includes songs and scales that can be performed with one or both upper limbs. Adaptable haptic assistance is available for more involved patients, and an algorithm adjusts task difficulty in proportion to patient performance.

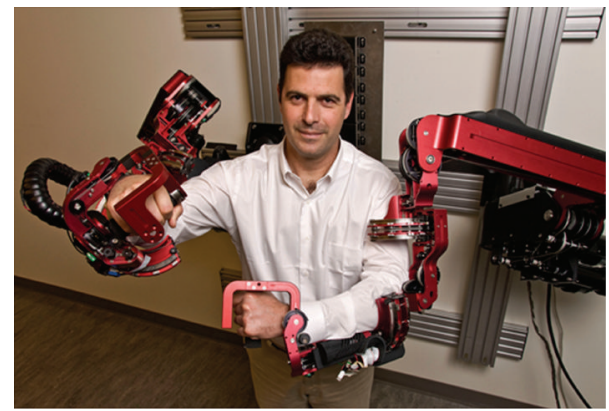

FIgURE 19: EXO-UL7. Reprinted with permission.

The Virtual Reality Piano was tested with four patients with chronic stroke [53]. All patients improved in performance time and key press accuracy, and 3 patients showed improvements in their ability to move each finger individually after 8-9 days of training for 90 minutes. Two patients improved on the Jebsen Test of Hand Function and 3 of the 4 patients improved on the WMFT.

The Virtual Reality Piano is not commercially available. The CyberGrasp is available at VRLOGIC GmbH, Dieburg, Germany and CyberGlove Systems LLC, San Jose, CA, USA.

3.2.14. EXO-UL7. The EXO-UL7 is a two-arm exoskeleton robot with 7 DOFs for each arm (see Figure 19). The seven single-axis revolute joints are responsible for shoulder abduction-adduction, flexion-extension and internalexternal rotation, elbow flexion-extension, and wrist 
TABLE 1: Summary of mechanical bilateral upper limb training devices.

\begin{tabular}{|c|c|c|c|c|}
\hline Name of device & $\begin{array}{l}\text { Movement of most } \\
\text { impaired upper } \\
\text { limb }\end{array}$ & Targeted part of the upper limb & $\begin{array}{l}\text { Device clinical investigation (patients } \\
\text { in phase post-stroke: acute, subacute, } \\
\text { or chronic) }\end{array}$ & $\begin{array}{l}\text { Commercially } \\
\text { available }\end{array}$ \\
\hline $\begin{array}{l}\text { BATRAC } \\
\text { (Tailwind) }\end{array}$ & Active & $\begin{array}{l}\text { Proximal } \\
\text { (shoulder and elbow) }\end{array}$ & $\begin{array}{l}\text { Yes } \\
2 \text { single group studies (chronic) } \\
{[20,22]} \\
1 \text { RCT (chronic) }[23]\end{array}$ & Yes \\
\hline $\begin{array}{l}\text { BATRAC } \\
\text { (modified) }\end{array}$ & Active & Distal (wrist) & $1 \mathrm{RCT}$ in progress (subacute) [21] & \\
\hline Reha-Slide Duo & Active & $\begin{array}{l}\text { Proximal } \\
\text { (shoulder and elbow) }\end{array}$ & No & Yes \\
\hline $\begin{array}{l}\text { Reha-Slide } \\
\text { (Nudelholz) }\end{array}$ & $\begin{array}{l}\text { Passive, assisted, } \\
\text { and active }\end{array}$ & $\begin{array}{l}\text { Proximal and distal } \\
\text { (shoulder, elbow, and wrist) }\end{array}$ & $\begin{array}{l}1 \text { case series (subacute) [25] } \\
1 \text { RCT (subacute) [26] }\end{array}$ & Yes \\
\hline $\begin{array}{l}\text { APBT } \\
\text { (the Rocker) }\end{array}$ & Passive & Distal (wrist) & $\begin{array}{l}1 \text { case series (subacute) [29] } \\
1 \text { single group study (subacute and } \\
\text { chronic) [27] } \\
1 \text { RCT (chronic) }[28]\end{array}$ & No \\
\hline Able-X & Active & $\begin{array}{l}\text { Proximal and distal } \\
\text { (shoulder, Elbow, and wrist) }\end{array}$ & 1 single group study (chronic) [30] & Yes \\
\hline
\end{tabular}

BATRAC: bilateral arm training with rhythmic auditory cueing; APBT: active-passive bimanual movement therapy; RCT: randomized clinical trial.

pronation-supination, flexion-extension, and radio-ulnar deviation [55]. Four six-axis force/torque sensors are attached to the upper arm, lower arm, the hand, and the tip of the exoskeleton for the human-machine interaction. Together with a control PC and a game PC, patients can manipulate virtual objects in video games while receiving haptic feedback [54]. The robot can be used for unilateral and bilateral training. In the unilateral mode the most impaired upper limb is supported with a weak assistive force toward the target. In the bilateral mode the desired joint angles are mirror symmetrically transmitted from the less impaired upper limb (master) to the most impaired upper limb (slave).

In a small RCT, 15 patients with chronic stroke were randomized over three groups: a unilateral robotic training group, a bilateral robotic training group, and a usual care group [54]. Only the results of both robotic training groups were reported. Both robotic training groups received two 90 -minute sessions of training a week, for 6 weeks. Each session a different combination of eight video games that interacted with the EXO-UL7 were played. The results showed no significant difference between the two robotic training groups in FMA score improvements. However, there was a significant difference in kinematic variables in favor of bilateral robotic training.

The EXO-UL7 is not commercially available.

A summary of the review for the mechanical bilateral upper limb training devices is provided in Table 1, while the robotic devices are summarized in Table 2 .

\section{Discussion}

In the foregoing, we reviewed the main characteristics of the bilateral upper limb training devices that have been proposed in the literature as well as their potential for clinical applications as a function of the currently available clinical evidence and commercial availability. Although the devices discussed in this paper are unlikely to represent the complete spectrum of bilateral upper limb training devices for poststroke rehabilitation, the paper is representative in that it covers the most prominent devices and provides an accurate, state-of-the-art overview of the potential of, and the difficulties involved in, the integration of mechanical and robotic devices in poststroke rehabilitation, including their availability.

Although the discussed devices have in common that they were all developed specifically for bilateral upper limb training or at least support bilateral upper limb training as one of the prominent training modes, they differ considerably in terms of their mechanical and electromechanical complexity. While the mechanical characteristics of some devices, like BATRAC (Tailwind), Reha-Slide and Reha-Slide Duo, are relatively simple, others involve complex forms of robotic control and sophisticated engineering solutions. The simpler devices are obviously easier to implement in rehabilitation, since they are relatively simple to operate and come at lower costs than the devices that use a lot of technical equipment for more precise measurement and movement control. Importantly, to the extent that solid clinical results are available for devices for bilateral upper arm training, no indications or trends are evident that the more intricate devices would have greater clinical efficacy than the simpler, less costly solutions.

As can be appreciated from Tables 1 and 2, the available evidence for the clinical efficacy of the bilateral upper arm training devices in poststroke rehabilitation is rather limited. No clinical results have been presented for 9 of the 20 devices discussed. Moreover, the evidence status of the clinical outcome measures reported for the other 8 devices is limited due to design limitations and other methodological 
TABLE 2: Summary of robotic bilateral upper limb training devices.

\begin{tabular}{|c|c|c|c|c|}
\hline $\begin{array}{l}\text { Name of } \\
\text { device }\end{array}$ & $\begin{array}{l}\text { Movement of } \\
\text { most impaired } \\
\text { upper limb }\end{array}$ & $\begin{array}{l}\text { Targeted part of } \\
\text { the upper limb }\end{array}$ & $\begin{array}{l}\text { Device clinical investigation } \\
\text { (patients in phase post-stroke: } \\
\text { acute, subacute, or chronic) }\end{array}$ & $\begin{array}{l}\text { Commercially } \\
\text { available }\end{array}$ \\
\hline $\mathrm{H}-\mathrm{O}-\mathrm{H}$ & Passive and assisted & Distal (wrist) & No & No \\
\hline Bimanual Lifting Rehabilitator & Passive and assisted & $\begin{array}{l}\text { Proximal } \\
\text { (shoulder and elbow) }\end{array}$ & No & No \\
\hline MIME & $\begin{array}{l}\text { Assisted (in bilateral } \\
\text { mode) }\end{array}$ & $\begin{array}{l}\text { Proximal } \\
\text { (shoulder and elbow) }\end{array}$ & $\begin{array}{l}1 \text { RCT (acute) [35] } \\
1 \text { RCT (subacute) [34] } \\
1 \text { RCT (chronic) [33] }\end{array}$ & No \\
\hline ARCMIME & $\begin{array}{l}\text { Assisted (in bilateral } \\
\text { mode) }\end{array}$ & $\begin{array}{l}\text { Proximal } \\
\text { (shoulder and elbow) }\end{array}$ & No & No \\
\hline Braccio di Ferro & Assisted and active & $\begin{array}{l}\text { Proximal } \\
\text { (shoulder and elbow) }\end{array}$ & No & No \\
\hline Bimanual Handlebar & Active & $\begin{array}{l}\text { Proximal } \\
\text { (shoulder and elbow) }\end{array}$ & No & No \\
\hline Bi-Manu Track & $\begin{array}{l}\text { Passive, assisted, and } \\
\text { active }\end{array}$ & $\begin{array}{l}\text { Distal } \\
\text { (elbow/forearm and wrist) }\end{array}$ & $\begin{array}{l}2 \text { RCTs (subacute) }[42,43] \\
3 \text { RCTs (chronic) }[44-46] \\
1 \text { RCT in progress (chronic) } \\
\text { (http://www.clinicaltrials.gov/, } \\
\text { NCT01525979) }\end{array}$ & Yes \\
\hline $\begin{array}{l}\text { Hand Robotic Rehabilitation } \\
\text { Device }\end{array}$ & $\begin{array}{l}\text { Passive, assisted, and } \\
\text { active }\end{array}$ & $\begin{array}{l}\text { Distal } \\
\text { (elbow/forearm and wrist) }\end{array}$ & No & No \\
\hline BFIAMT & $\begin{array}{l}\text { Passive, assisted, and } \\
\text { active }\end{array}$ & $\begin{array}{l}\text { Proximal } \\
\text { (shoulder and elbow) }\end{array}$ & $\begin{array}{l}1 \text { single group study } \\
\text { (chronic) }[48]\end{array}$ & No \\
\hline $\begin{array}{l}\text { Bimanual Coordinate Training } \\
\text { System }\end{array}$ & $\begin{array}{l}\text { Passive, assisted, and } \\
\text { active }\end{array}$ & Proximal (elbow) & No & No \\
\hline Driver's SEAT & $\begin{array}{l}\text { Passive, assisted, and } \\
\text { active }\end{array}$ & $\begin{array}{l}\text { Proximal } \\
\text { (shoulder and elbow) }\end{array}$ & No & No \\
\hline $\begin{array}{l}\text { Adaptive Bimanual Robotic } \\
\text { Training }\end{array}$ & $\begin{array}{l}\text { Active (with passive } \\
\text { gravity compensation) }\end{array}$ & $\begin{array}{l}\text { Proximal } \\
\text { (shoulder and elbow) }\end{array}$ & No & No \\
\hline Virtual Reality Piano & Assisted and active & $\begin{array}{l}\text { Distal } \\
\text { (wrist and fingers) }\end{array}$ & $\begin{array}{l}1 \text { single group } \\
\text { study (chronic) [53] }\end{array}$ & $\begin{array}{l}\text { Virtual Reality } \\
\text { Piano: no } \\
\text { CyberGrasp: yes } \\
\end{array}$ \\
\hline EXO-UL7 & Assisted & $\begin{array}{l}\text { Proximal and distal } \\
\text { (shoulder, elbow, Forearm, } \\
\text { and wrist) }\end{array}$ & 1 RCT (chronic) [54] & No \\
\hline
\end{tabular}

H-O-H: hand-object-hand; MIME: Mirror Image Movement Enabler; ARCMIME: Applied Resources Corp. Mirror Image Movement Enabler; BFIAMT: bilateral force-induced isokinetic arm movement Trainer; RCT: randomized clinical trial.

shortcomings. Among these are the following. First of all, only some RCTs have been conducted to test the efficacy of the discussed devices. According to the commonly accepted hierarchy of evidence, the most reliable evidence comes from meta-analyses in systematic reviews, followed by evidence from RCTs, cohort studies, and then case studies. Positive results from the case studies and cohort studies discussed in this paper only provide an indication that a particular device may be beneficial in rehabilitation. In many cases, a comparison with other relevant forms of treatment is lacking so that it is impossible to make any statements about the relative superiority or inferiority of training on a particular device relative to other interventions. Second, some of the reported RCTs may be biased because they did not control for the amount of training. For example, the amount of time spent in training by the APBT group was larger than by the control group [28], and the number of repetitions of wrist movements in the Bi-Manu-Track group far exceeded the number of repetitions in the electrical stimulation group [41]. Of course, admittedly, this discrepancy between the groups is tied to one of the advantages offered by mechanical or robotic training devices, namely that the number of movement repetitions can be considerably increased compared to conventional treatment programs. Third, in several studies in which positive clinical outcomes were found, it is impossible to ascribe the observed effects to bilateral training per se, as the training protocols also involved unilateral training. Likewise, it is impossible to tell whether the effects were due to practicing proximal or more distal parts of the most affected arm, to active, passive, or assisted training of the most impaired limb, or to performing in-phase or anti-phase coordination patterns, because none of these aspects were systematically controlled for in the design of the studies in question. 
Based on the discussed clinical results and results from prior meta-analyses [13-18], bilateral upper limb training (with or without training devices) appears to be at least as effective as alternative forms of treatment, with the added advantage that training devices allow an increase in training intensity and frequency as well as the opportunity to train independently [1-4]. However, much more research is needed than has been conducted thus far in order to tailor treatments to the specific needs and characteristics of individual patients. First, there is a definite need for RCT's aimed specifically and systematically at testing various aspects of bilateral training: bimanual versus unimanual, proximal versus distal, active versus passive or assisted movement, and their efficacy in patients with (sub)acute versus chronic stroke. Second, it is even more important to know exactly what it is that patients learn from bilateral upper limb training and what mechanisms are underlying this learning process. This means that measuring changes in clinical outcomes is not sufficient, but that measures of neural reorganization (see also [23]), kinematics, and timing also have to be incorporated, as, for example, in the ULTRAstroke trial [21]. As it stands, there is very little we can conclude vis-à-vis these aspects from the existing literature.

In conclusion, one may conclude that the principle of bilateral training, and the promise it holds for the rehabilitation of the motor function of the upper limbs, has sparked not only theoretically motivated research in this area, but also various promising innovations in mechanical and robotic devices aimed specifically, or at least partially, at accommodating bilateral training of the upper limbs. However, initial clinical results are not yet of such caliber that the devices in question and the concepts on which they are based are firmly established. It is rather the case that the initial clinical outcomes do not rule out the possibility that concept of bilateral training and the accompanied mechanical and robotic devices may provide a useful extension of currently available forms of therapy. However, to actually demonstrate their value, more research with adequate experimental, dose-matched designs and sufficient statistical power is required. As it stands, one should avoid the proliferation of technological devices that are not sufficiently evidence based and insure that technological advances become and stay aligned with conceptual and empirical developments.

\section{Acknowledgments}

The authors would like to thank Sergei V. Adamovich, Kris Appel, Eric Aßmann, Charles G. Burgar, Maura Casadio, Gerard G. Fluet, Juha M. Hijmans, Machiel van der Loos, Peter S. Lum, Alireza Mirbaghery, Eshan Rashedi, Jacob Rosen, Mary Ellen Stoykov, Fong-Chin Su, and Matic Trlep for providing photos.

\section{References}

[1] G. Kwakkel, B. J. Kollen, and H. I. Krebs, "Effects of robotassisted therapy on upper limb recovery after stroke: a systematic review," Neurorehabilitation and Neural Repair, vol. 22, no. 2, pp. 111-121, 2008.
[2] J. Mehrholz, T. Platz, J. Kugler, and M. Pohl, "Electromechanical and robot-assisted arm training for improving arm function and activities of daily living after stroke," Cochrane Database of Systematic Reviews, no. 4, Article ID CD006876, 2008.

[3] G. B. Prange, M. J. A. Jannink, C. G. M. GroothuisOudshoorn, H. J. Hermens, and M. J. IJzerman, "Systematic review of the effect of robot-aided therapy on recovery of the hemiparetic arm after stroke," Journal of Rehabilitation Research and Development, vol. 43, no. 2, pp. 171-183, 2006.

[4] A. A. Timmermans, H. A. Seelen, R. D. Willmann, and H. Kingma, "Technology-assisted training of arm-hand skills in stroke: concepts on reacquisition of motor control and therapist guidelines for rehabilitation technology design," Journal of NeuroEngineering and Rehabilitation, vol. 6, no. 1, article 1, 2009.

[5] R. Kumar, E. J. Metter, A. J. Mehta, and T. Chew, "Shoulder pain in hemiplegia: the role of exercise," American Journal of Physical Medicine and Rehabilitation, vol. 69, no. 4, pp. 205208, 1990.

[6] M. H. Mudie and T. A. Matyas, "Upper extremity retraining following stroke: effects of bilateral practice," Neurorehabilitation and Neural Repair, vol. 10, no. 3, pp. 167-184, 1996.

[7] M. H. Mudie and T. A. Matyas, "Can simultaneous bilateral movement involve the undamaged hemisphere in reconstruction of neural networks damaged by stroke?" Disability and Rehabilitation, vol. 22, no. 1-2, pp. 23-37, 2000.

[8] L. Cohen, "Synchronous bimanual movements performed by homologous and non-homologous muscles," Perceptual and Motor Skills, vol. 32, no. 2, pp. 639-644, 1971.

[9] J. A. S. Kelso, "Phase transitions and critical behavior in human bimanual coordination," American Journal of Physiology, vol. 246, no. 6, pp. R1000-R1004, 1984.

[10] J. A. S. Kelso, D. L. Southard, and D. Goodman, "On the coordination of two-handed movements," Journal of Experimental Psychology, vol. 5, no. 2, pp. 229-238, 1979.

[11] A. Ridderikhoff, C. E. Peper, and P. J. Beek, "Unraveling interlimb interactions underlying bimanual coordination," Journal of Neurophysiology, vol. 94, no. 5, pp. 3112-3125, 2005.

[12] S. P. Swinnen, "Intermanual coordination: from behavioural principles to neural-network interactions," Nature Reviews Neuroscience, vol. 3, no. 5, pp. 350-361, 2002.

[13] J. H. Cauraugh, N. Lodha, S. K. Naik, and J. J. Summers, "Bilateral movement training and stroke motor recovery progress: a structured review and meta-analysis," Human Movement Science, vol. 29, no. 5, pp. 853-870, 2010.

[14] K. C. Stewart, J. H. Cauraugh, and J. J. Summers, "Bilateral movement training and stroke rehabilitation: a systematic review and meta-analysis," Journal of the Neurological Sciences, vol. 244, no. 1-2, pp. 89-95, 2006.

[15] C. P. Latimer, J. Keeling, B. Lin, M. Henderson, and L. A. Hale, "The impact of bilateral therapy on upper limb function after chronic stroke: a systematic review," Disability and Rehabilitation, vol. 32, no. 15, pp. 1221-1231, 2010.

[16] F. Coupar, A. Pollock, F. van Wijck, J. Morris, and P. Langhorne, "Simultaneous bilateral training for improving arm function after stroke," Cochrane Database of Systematic Reviews, vol. 4, Article ID CD006432, 2010.

[17] P. Langhorne, F. Coupar, and A. Pollock, "Motor recovery after stroke: a systematic review," The Lancet Neurology, vol. 8, no. 8, pp. 741-754, 2009.

[18] A. E. Q. Van Delden, C. E. Peper, P. J. Beek, and G. Kwakkel, "Unilateral versus bilateral upper limb exercise therapy after 
stroke: a systematic review," Journal of Rehabilitation Medicine, vol. 44, no. 2, pp. 106-117, 2012.

[19] P. S. Lum, D. J. Reinkensmeyer, and S. L. Lehman, "Robotic assist devices for bimanual physical therapy: preliminary experiments," IEEE Transactions on Rehabilitation Engineering, vol. 3, no. 2, pp. 185-191, 1993.

[20] J. Whitall, S. M. Waller, K. H. C. Silver, and R. F. Macko, "Repetitive bilateral arm training with rhythmic auditory cueing improves motor function in chronic hemiparetic stroke," Stroke, vol. 31, no. 10, pp. 2390-2395, 2000.

[21] A. E. Q. Van Delden, C. E. Peper, J. Harlaar et al., "Comparing unilateral and bilateral upper limb training: the ULTRA-stroke program design," BMC Neurology, vol. 9, article 57, 2009.

[22] L. G. Richards, C. R. Senesac, S. B. Davis, M. L. Woodbury, and S. E. Nadeau, "Bilateral arm training with rhythmic auditory cueing in chronic stroke: not always efficacious," Neurorehabilitation and Neural Repair, vol. 22, no. 2, pp. 180 184, 2008.

[23] J. Whitall, S. McCombe Waller, J. D. Sorkin et al., "Bilateral and unilateral arm training improve motor function through differing neuroplastic mechanisms: a single-blinded randomized controlled trial," Neurorehabilitation and Neural Repair, vol. 25, no. 2, pp. 118-129, 2011.

[24] R. Buschfort, J. Brocke, A. Heß, C. Werner, A. Waldner, and S. Hesse, "Arm studio to intensify upper limb rehabilitation after stroke: concept, acceptance, utilization and preliminary clinical results," Journal of Rehabilitation Medicine, vol. 42, no. 4, pp. 310-314, 2010.

[25] S. Hesse, H. Schmidt, C. Werner, C. Rybski, U. Puzich, and A. Bardeleben, "A new mechanical arm trainer to intensify the upper limb rehabilitation of severely affected patients after stroke: design, concept and first case series," Europa Medicophysica, vol. 43, no. 4, pp. 463-468, 2007.

[26] S. Hesse, C. Werner, M. Pohl, J. Mehrholz, U. Puzich, and H. I. Krebs, "Mechanical arm trainer for the treatment of the severely affected arm after a stroke: a single-blinded randomized trial in two centers," American Journal of Physical Medicine and Rehabilitation, vol. 87, no. 10, pp. 779-788, 2008.

[27] J. W. Stinear and W. D. Byblow, "Rhythmic bilateral movement training modulates corticomotor excitability and enhances upper limb motricity poststroke: a pilot study," Journal of Clinical Neurophysiology, vol. 21, no. 2, pp. 124-131, 2004.

[28] C. M. Stinear, P. A. Barber, J. P. Coxon, M. K. Fleming, and W. D. Byblow, "Priming the motor system enhances the effects of upper limb therapy in chronic stroke," Brain, vol. 131, no. 5, pp. 1381-1390, 2008.

[29] M. E. Stoykov and J. W. Stinear, "Active-passive bilateral therapy as a priming mechanism for individuals in the subacute phase of post-stroke recovery: a feasibility study," American Journal of Physical Medicine and Rehabilitation, vol. 89, no. 11, pp. 873-878, 2010.

[30] J. M. Hijmans, L. A. Hale, J. A. Satherley, N. J. McMillan, and M. J. King, "Bilateral upper-limb rehabilitation after stroke using a movement-based game controller," Journal of Rehabilitation Research and Development, vol. 48, no. 8, pp. 1005-1014, 2011.

[31] P. S. Lum, S. L. Lehman, and D. J. Reinkensmeyer, "Bimanual lifting rehabilitator: an adaptive machine for therapy of stroke patients," IEEE Transactions on Rehabilitation Engineering, vol. 3, no. 2, pp. 166-173, 1995.

[32] C. G. Burgar, P. S. Lum, P. C. Shor, and H. F. M. Van Der Loos, "Development of robots for rehabilitation therapy: the Palo Alto VA/Stanford experience," Journal of Rehabilitation Research and Development, vol. 37, no. 6, pp. 663-673, 2000.
[33] P. S. Lum, C. G. Burgar, P. C. Shor, M. Majmundar, and M. Van der Loos, "Robot-assisted movement training compared with conventional therapy techniques for the rehabilitation of upper-limb motor function after stroke," Archives of Physical Medicine and Rehabilitation, vol. 83, no. 7, pp. 952-959, 2002.

[34] P. S. Lum, C. G. Burgar, M. Van Der Loos, P. C. Shor, M. Majmundar, and R. Yap, "MIME robotic device for upperlimb neurorehabilitation in subacute stroke subjects: a followup study," Journal of Rehabilitation Research and Development, vol. 43, no. 5, pp. 631-642, 2006.

[35] C. G. Burgar, P. S. Lum, A. M. Erika Scremin et al., "Robotassisted upper-limb therapy in acute rehabilitation setting following stroke: department of veterans affairs multisite clinical trial," Journal of Rehabilitation Research and Development, vol. 48, no. 4, pp. 445-458, 2011.

[36] R. M. Mahoney, H. F. M. Van der Loos, P. S. Lum, and C. Burgar, "Robotic stroke therapy assistant," Robotica, vol. 21, no. 1, pp. 33-44, 2003.

[37] M. Casadio, V. Sanguineti, P. G. Morasso, and V. Arrichiello, "Braccio di Ferro: a new haptic workstation for neuromotor rehabilitation," Technology and Health Care, vol. 14, no. 3, pp. 123-142, 2006.

[38] V. Sanguineti, M. Casadio, E. Vergaro, V. Squeri, P. Giannoni, and P. G. Morasso, "Robot therapy for stroke survivors: proprioceptive training and regulation of assistance," Studies in Health Technology and Informatics, vol. 145, pp. 126-142, 2009.

[39] V. Squeri, M. Casadio, E. Vergaro, P. Giannoni, P. Morasso, and V. Sanguineti, "Bilateral robot therapy based on haptics and reinforcement learning: feasibility study of a new concept for treatment of patients after stroke," Journal of Rehabilitation Medicine, vol. 41, no. 12, pp. 961-965, 2009.

[40] M. Trlep, M. Mihelj, and M. Munih, "Skill transfer from symmetric and asymmetric bimanual training using a robotic system to single limb performance," Journal of NeuroEngineering and Rehabilitation, vol. 9, article 43, 2012.

[41] S. Hesse, G. Schulte-Tigges, M. Konrad, A. Bardeleben, and C. Werner, "Robot-assisted arm trainer for the passive and active practice of bilateral forearm and wrist movements in hemiparetic subjects," Archives of Physical Medicine and Rehabilitation, vol. 84, no. 6, pp. 915-920, 2003.

[42] S. Hesse, C. Werner, M. Pohl, S. Rueckriem, J. Mehrholz, and M. L. Lingnau, "Computerized arm training improves the motor control of the severely affected arm after stroke: a single-blinded randomized trial in two centers," Stroke, vol. 36, no. 9, pp. 1960-1966, 2005.

[43] S. Hesse, A. Waldner, J. Mehrholz, C. Tomelleri, M. Pohl, and C. Werner, "Combined transcranial direct current stimulation and robot-assisted arm training in subacute stroke patients: an exploratory, randomized multicenter trial," Neurorehabilitation and Neural Repair, vol. 25, no. 9, pp. 838-846, 2011.

[44] W.-W. Liao, C.-Y. Wu, Y.-W. Hsieh, K.-C. Lin, and W.-Y. Chang, "Effects of robot-assisted upper limb rehabilitation on daily function and real-world arm activity in patients with chronic stroke: a randomized controlled trial," Clinical Rehabilitation, vol. 26, no. 2, pp. 111-120, 2012.

[45] C.-L. Yang, K.-C. Lin, H.-C. Chen, C.-Y. Wu, and C.-L. Chen, "Pilot comparative study of unilateral and bilateral robotassisted training on upper-extremity performance in patients with stroke," American Journal of Occupational Therapy, vol. 66, no. 2, pp. 198-206, 2012.

[46] C. Wu, C. Yang, L. Chuang et al., "Effect of therapist-based versus robot-assisted bilateral arm training on motor control, functional performance, and quality of life after chronic 
stroke: a clinical trial," Physical Therapy, vol. 92, no. 8, pp. 1006-1016, 2012.

[47] E. Rashedi, A. Mirbagheri, B. Taheri, F. Farahmand, G. Vossoughi, and M. Parnianpour, "Design and development of a hand robotic rehabilitation device for post stroke patients," in Proceedings of the 31st Annual International Conference of the IEEE Engineering in Medicine and Biology Society: Engineering the Future of Biomedicine (EMBC '09), pp. 50265029, September 2009.

[48] J. J. Chang, W. L. Tung, W. L. Wu, M. H. Huang, and F. C. Su, "Effects of robot-aided bilateral force-induced isokinetic arm training combined with conventional rehabilitation on arm motor function in patients with chronic stroke," Archives of Physical Medicine and Rehabilitation, vol. 88, no. 10, pp. 1332 1338, 2007.

[49] C. Li, Y. Inoue, T. Lju, K. Shibata, and K. Oka, "A new master-slave control method for implementing force sensing and energy recycling in a bilateral arm training robot," International Journal of Innovative Computing, Information and Control, vol. 7, no. 1, pp. 471-485, 2011.

[50] M. J. Johnson, H. F. M. Van der Loos, C. G. Burgar, P. Shor, and L. J. Leifer, "Design and evaluation of Driver's SEAT: a car steering simulation environment for upper limb stroke therapy," Robotica, vol. 21, no. 1, pp. 13-23, 2003.

[51] M. J. Johnson, H. F. M. Van Der Loos, C. G. Burgar, P. Shor, and L. J. Leifer, "Experimental results using force-feedback cueing in robot-assisted stroke therapy," IEEE Transactions on Neural Systems and Rehabilitation Engineering, vol. 13, no. 3, pp. 335-348, 2005.

[52] M. Trlep, M. Mihelj, U. Puh, and M. Munih, "Rehabilitation robot with patient-cooperative control for bimanual training of hemiparetic subjects," Advanced Robotics, vol. 25, no. 15, pp. 1949-1968, 2011.

[53] S. V. Adamovich, A. Mathai, G. G. Fluet, Q. Qiu, J. Lewis, and A. S. Merians, "Design of a complex virtual reality simulation to train finger motion for persons with hemiparesis: a proof of concept study," Journal of NeuroEngineering and Rehabilitation, vol. 6, no. 1, article 28, 2009.

[54] H. Kim, L. Miller, I. Fedulow et al., "Kinematic data analysis for post stroke patients following bilateral versus unilateral rehabilitation with an upper limb wearable robotic system," IEEE Transactions on Neural Systems and Rehabilitation Engineering. In press.

[55] J. Rosen and J. C. Perry, "Upper limb powered exoskeleton," International Journal of Humanoid Robotics, vol. 4, no. 3, pp. 529-548, 2007. 


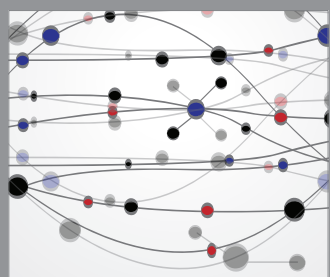

The Scientific World Journal
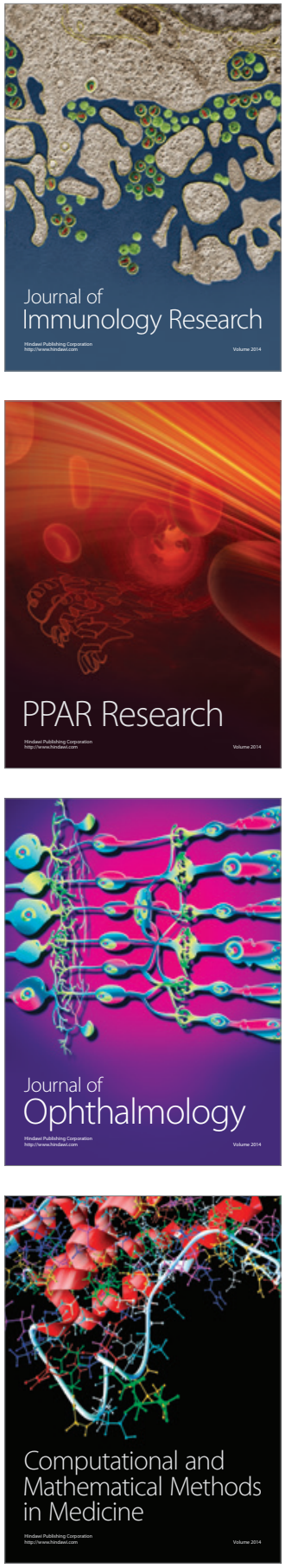

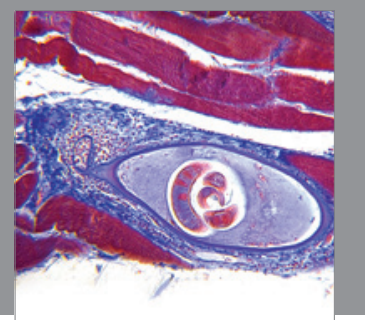

Gastroenterology

Research and Practice
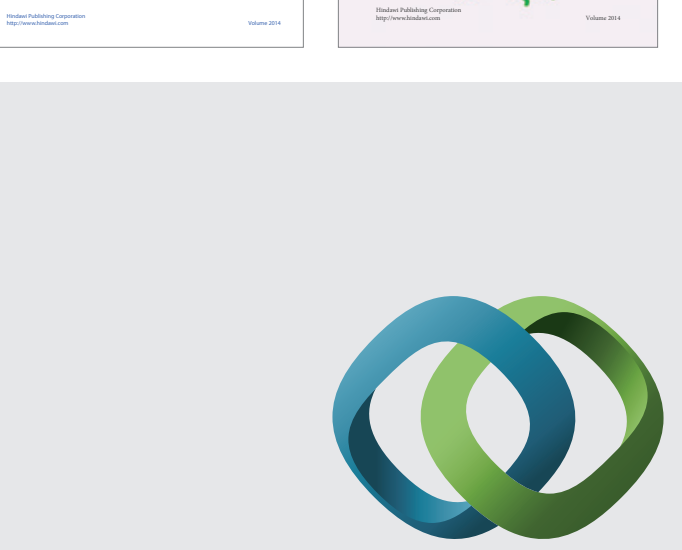

\section{Hindawi}

Submit your manuscripts at

http://www.hindawi.com
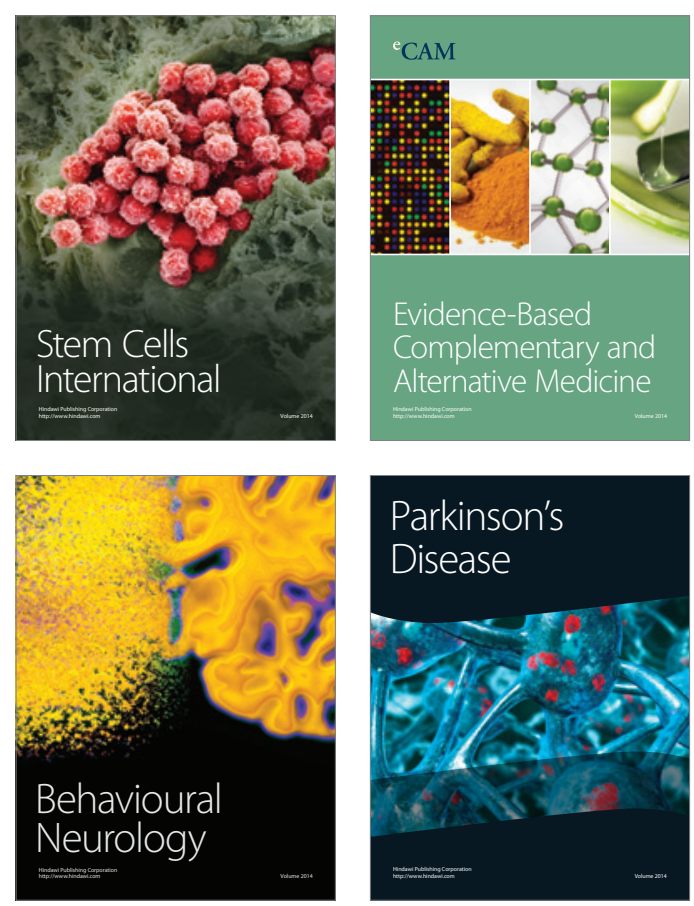

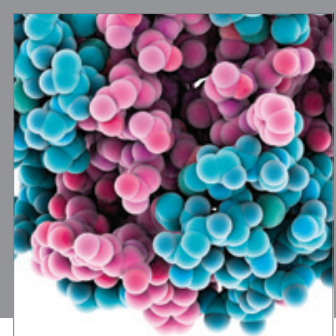

Journal of
Diabetes Research

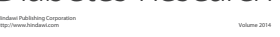

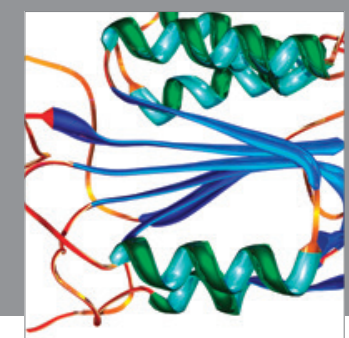

Disease Markers
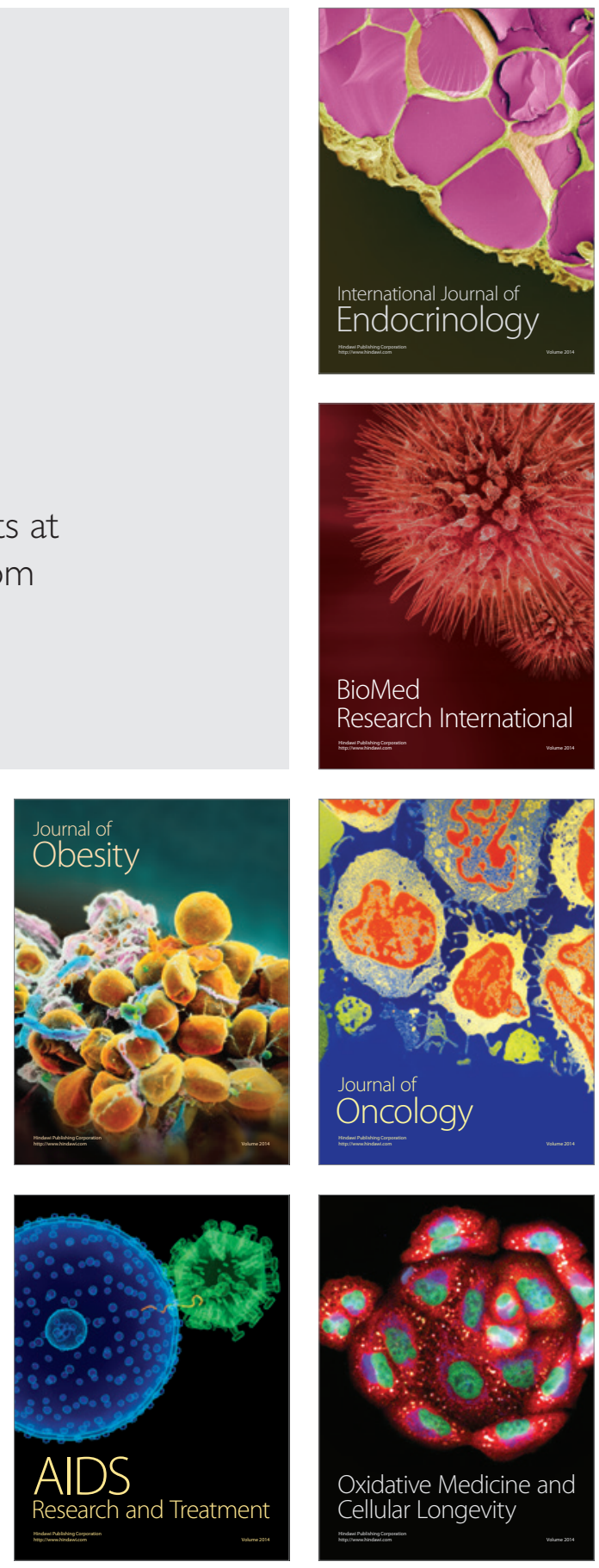